\title{
Advanced Three Waveguide Sagnac Loop Reflectors for High Performance Optical Filters
}

\author{
David J. Moss, Fellow, IEEE, Fellow, OSA
}

\begin{abstract}
We theoretically investigate advanced multi-functional integrated photonic filters formed by three waveguide coupled Sagnac loop reflectors (3WC-SLRs). By tailoring the coherent mode interference, the spectral response of the 3WC-SLR resonators is engineered to achieve diverse filtering functions with high performance. These include optical analogues of Fano resonances that yield ultrahigh spectral extinction ratios (ERs) and slope rates, resonance mode splitting with high ERs and low free spectral ranges, and classical Butterworth, Bessel, Chebyshev, and elliptic filters. A detailed analysis of the impact of the structural parameters and fabrication tolerances is provided to facilitate device design and optimization. The requirements for practical applications are also considered. These results theoretically verify the effectiveness of using $3 \mathrm{WC}$ SLR resonators as multi-functional integrated photonic filters for flexible spectral engineering in diverse applications.
\end{abstract}

Keywords-Integrated optics, resonators, Fano resonance, mode splitting, classical filters.

\section{INTRODUCTION}

$\mathrm{I}_{\mathrm{sen}}^{\mathrm{N}}$ concert with the advance of complementary metal-oxidesemiconductor (CMOS) fabrication technologies, particularly as applied to photonic integrated circuits, integrated photonic resonators have become key building blocks $[1,2]$. With narrowband wavelength selectivity, strong resonance field enhancement, and versatile filter shapes, these micro/nano-scale devices have been widely used for diverse applications including filters, lasers, modulators, buffers, switches, sensors, and signal processors [2-10]. and have also been applied to phase-sensitive all-pass filters [11-15]. Integrated ring resonators, particularly in CMOS compatible platforms, have also formed the basis for Kerr microcombs, [16-31] that have experienced wide applications to microwave photonics, [32-55] data communications [56-58] and neural networks [59, 60], and quantum optics [61- 69]. Photonic crystal cavities form a key class of integrated resonator that have been widely investigated [70-76]. For all of these devices, CMOS compatible platforms have proven to be indispensable [77 - 88] for their low loss, reproducibility and manufacturability, particularly for nonlinear optical applications where they have out-performed silicon [89-103] and even chalcogenide glass [104-117] and even other materials [118-120] because of their low nonlinear absorption [121-123]. This has been a motivation for integrating novel

The authors are with the Optical Sciences Centre, Swinburne University of Technology, Hawthorn, VIC 3122, Australia (e-mail: dmoss@swin.edu.au).
2D materials onto CMOS platforms, to increase their nonlinear performance [124 - 130].

Fano resonances are a fundamental physical phenomenon featuring an asymmetric resonant lineshape profile induced by interference between a discrete localized state and a continuum state [131-134]. It was first discovered in the absorption spectra of noble gases and later on has been extended to a much wider scope $[135,136]$. In recent years, various types of photonic resonators have been designed to realize optical analogues of Fano resonances, which have attracted great interest and found wide applications in optical switching, sensing, light focusing beyond the diffraction limit, topological optics, data storage, and many others [[131, 133, 137-139].

Resonance mode splitting is a fundamental phenomenon in photonic resonators that occurs when two or more mutually coupled modes co-exist in the same resonant cavity [140, 141]. It can achieve a reduced free spectral range (FSR) and an increased quality (Q) factor while maintaining a small physical cavity length, thus yielding a compact device footprint, low power consumption, and versatile filter shapes for dense-wavelength division-multiplexing (DWDM) and microwave photonics applications $[142,143]$. Recently, many applications based on mode-split resonators have been demonstrated, such as optical buffering [144], dispersion compensation [145,146], signal multicasting [147, 148], differential equation solving [149], microwave signal generation [150], and sensing [151].

Butterworth, Bessel, Chebyshev, and elliptic filters are classical filters that model and govern signal filtering and processing in communications and computing systems [152154]. These fundamental filters are broadly used in applications such as noise reduction, spectral analysis, and signal synthesis [155-158]. Photonic resonators offer a powerful solution to realize these filters in optical domain [159-161]. With much broader operation bandwidth than their electronic counterparts, these optical filters play an important role in high-speed optical communications and information processing [162].

To realize Fano-resonances, resonance mode splitting, and classical filters based on integrated photonic resonators could offer competitive advantages including compact footprint, high scalability, high stability, and mass producibility for practical applications. Recently, we investigated multifunctional integrated photonic filters based on cascaded Sagnac loop reflectors (SLRs) [163] and two waveguide coupled Sagnac loop reflectors (2WC-SLRs) [164] formed by 
self-coupled silicon-on-insulator (SOI) nanowires. Here, we theoretically investigate more complex device structures including three waveguide coupled Sagnac loop reflectors (3WC-SLRs) that yield greatly enhanced performance together with more versatile filtering functions. We tailor coherent mode interference in the $3 \mathrm{WC}-\mathrm{SLR}$ resonators to achieve versatile filter shapes with high performance, including optical analogues of Fano resonances with ultrahigh extinction ratios (ERs) and slope rates (SRs), resonance mode splitting with high ERs and low FSRs, and classical Butterworth, Bessel, Chebyshev, and elliptic filters. A detailed analysis of the impact of the structural parameters and fabrication tolerances is provided. The requirements for practical applications are also considered in our design and achieved for the proposed devices. These results highlight the strong potential of $3 \mathrm{WC}-\mathrm{SLR}$ resonators for flexible spectral engineering in diverse applications such as optical filtering, switching, and sensing.

\section{Device Configuration}

Figure 1 illustrates schematic configurations of the $3 \mathrm{WC}$ -
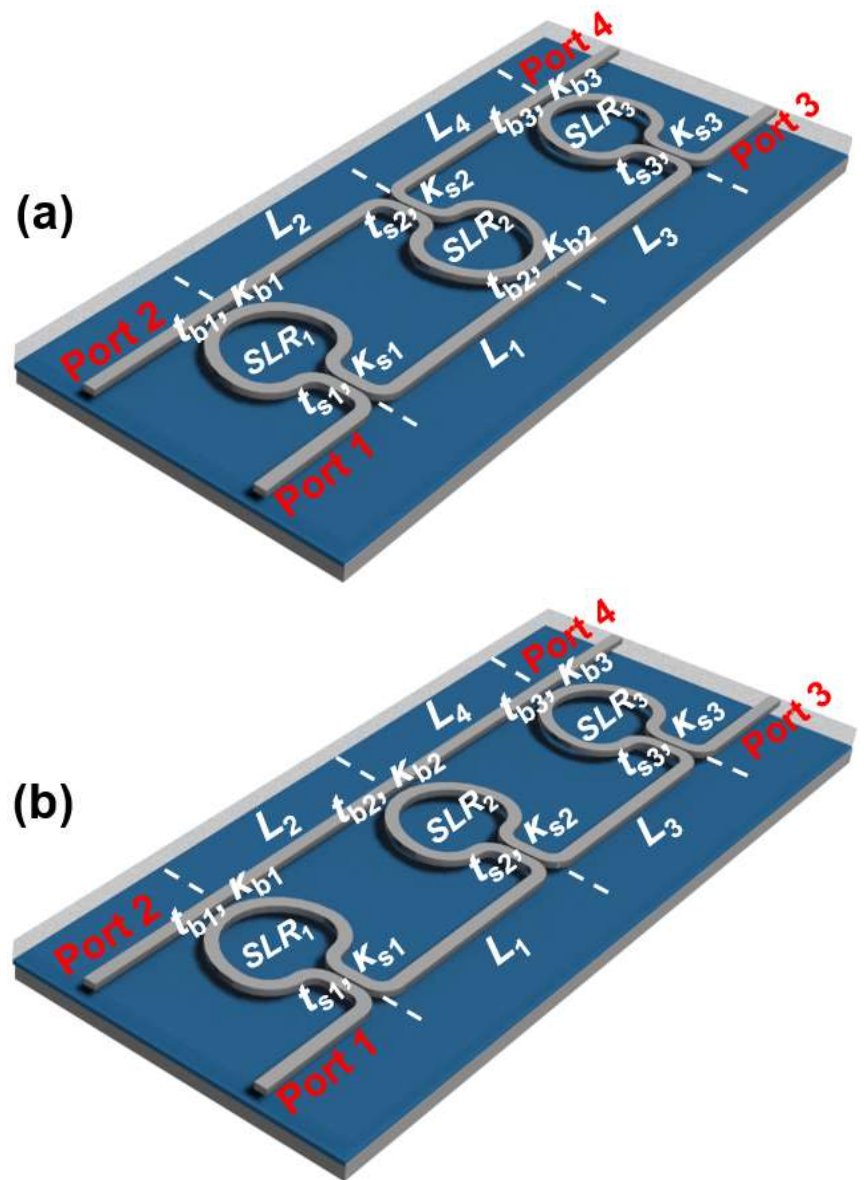

Fig. 1. Schematic configuration of (a) zig-zag and (b) parallel $3 \mathrm{WC}-\mathrm{SLR}$ resonators consisting of three SLRs $\left(S L R_{1}, S L R_{2}\right.$, and $\left.S L R_{3}\right)$, respectively. The definitions of $t_{\mathrm{si}}(i=1,2,3), t_{\mathrm{b} i}(i=1,2,3), L_{\mathrm{SLR} i}(i=1,2,3)$, and $L_{i}(i=1,2$, $3,4)$ are given in Table I.

SLR resonators. Two types of $3 \mathrm{WC}-\mathrm{SLR}$ resonators are investigated. The first one in Fig. 1(a) consists of three inversely coupled SLRs and is termed a zig-zag 3WC-SLR resonator, while the second one in Fig. 1(b) consists of three parallel Sagnac loop reflectors (SLRs) coupled to a top bus waveguide, and is termed a parallel 3WC-SLR resonator. In both $3 \mathrm{WC}-\mathrm{SLR}$ resonators, the bus waveguides between the SLRs introduce additional feedback paths for coherent optical mode interference, yielding greatly improved flexibility for engineering the spectral response. To study the 3WC-SLR resonators based on the scattering matrix method [163-165], the waveguide and coupler parameters are defined in Table I. To simplify the comparison, we assume that the three SLRs are identical in each $3 \mathrm{WC}-\mathrm{SLR}$ resonator, i.e., $L_{\mathrm{SLR} 1}=L_{\mathrm{SLR} 2}=$ $L_{\mathrm{SLR} 3}=L_{\mathrm{SLR}}, t_{\mathrm{s} 1}=t_{\mathrm{s} 2}=t_{\mathrm{s} 3}=t_{\mathrm{s}}$, and $t_{\mathrm{b} 1}=t_{\mathrm{b} 2}=t_{\mathrm{b} 3}=t_{\mathrm{b}}$.

The zig-zag $3 \mathrm{WC}-\mathrm{SLR}$ resonator is equivalent to two cascaded Mach-Zehnder interferometers (MZIs, which is a finite-impulse-response (FIR) filter) and a Fabry-Perot cavity (which is an infinite-impulse-response (IIR) filter) when $t_{\mathrm{s}}=1$ and $t_{\mathrm{b}}=1$, respectively. On the other hand, the parallel $3 \mathrm{WC}$ SLR resonator is equivalent to two cascaded MZIs and three cascaded SLRs (which is an IIR filter) when $t_{\mathrm{s}}=1$ and $t_{\mathrm{b}}=1$, respectively. When $t_{\mathrm{s}} \neq 1$ and $t_{\mathrm{b}} \neq 1$, both types of filters can be considered to be hybrid, consisting of both FIR and IIR filter elements that allows more versatile coherent mode interference induced by mutual interaction. As compared with the 2WC-SLR resonators [164], the 3WC-SLR resonators have an extra SLR and additional feedback paths that introduce more complex coherent mode interference, which can lead to enhanced filter performance and versatility. The freedom in designing the reflectivity of the SLRs (i.e., $t_{\mathrm{s}}$ ), the coupling strength between the SLRs and connecting bus waveguides (i.e., $t_{\mathrm{b}}$ ), and the waveguide lengths (i.e., $L_{\mathrm{SLR}}$ and $L_{i}$ ) enables flexible spectral engineering based on the $3 \mathrm{WC}$ SLR resonators, which can lead to diverse filtering functions.

In the following sections, mode interference in the $3 \mathrm{WC}$ SLR resonators is tailored to achieve high-performance filtering functions, including optical analogues of Fano resonances (Section III), resonance mode splitting (Section IV), and classical Butterworth, Chebyshev, Bessel, and elliptic filters (Section V). In our design, we use values obtained from our previously fabricated SOI devices $[163,166]$ for the waveguide group index $\left(n_{\mathrm{g}}=4.3350\right.$, transverse electric (TE) mode) and the propagation loss $\left(\alpha=55 \mathrm{~m}^{-1}\right.$, i.e., $\left.2.4 \mathrm{~dB} / \mathrm{cm}\right)$. The devices are designed based on but not limited to the SOI integrated platform. 
TABLE I

DEFINITIONS OF STRUCTURAL PARAMETERS OF THE 3WC-SLR RESONATORS

\begin{tabular}{|c|c|c|c|c|}
\hline Waveguides & Length & \multicolumn{2}{|c|}{$\begin{array}{c}\text { Transmission } \\
\text { factor }^{\mathrm{a}}\end{array}$} & Phase shift ${ }^{b}$ \\
\hline $\begin{array}{c}\text { Bus waveguides between } \\
\text { SLRs }(i=1,2,3,4)\end{array}$ & $L_{i}$ & \multicolumn{2}{|c|}{$a_{i}$} & $\varphi_{i}$ \\
\hline $\begin{array}{c}\text { Sagnac loop in } S L R_{i} \\
(i=1,2,3)\end{array}$ & $L_{\mathrm{SLR} i}$ & \multicolumn{2}{|c|}{$a_{\mathrm{s} i}$} & $\varphi_{\mathrm{s} i}$ \\
\hline Directional couplers & \multicolumn{2}{|c|}{$\begin{array}{c}\text { Field transmission } \\
\text { coefficient }^{c}\end{array}$} & \multicolumn{2}{|c|}{$\begin{array}{l}\text { Field cross-coupling } \\
\text { coefficient }^{c}\end{array}$} \\
\hline $\begin{array}{c}\text { Coupler in } S L R_{i} \\
(i=1,2,3)\end{array}$ & \multicolumn{2}{|c|}{$t_{\mathrm{si}}$} & \multicolumn{2}{|r|}{$k_{\mathrm{s} i}$} \\
\hline $\begin{array}{c}\text { Coupler between } S L R_{i} \\
\text { and bus waveguide } \\
(i=1,2,3)\end{array}$ & \multicolumn{2}{|c|}{$t_{\mathrm{b} i}$} & \multicolumn{2}{|r|}{$k_{\mathrm{b} i}$} \\
\hline
\end{tabular}

a $a_{i}=\exp \left(-\alpha L_{i} / 2\right), a_{\mathrm{s} i}=\exp \left(-\alpha L_{\mathrm{SLR} i} / 2\right), \alpha$ is the power propagation loss factor.

${ }^{\mathrm{b}} \varphi_{i}=2 \pi n_{\mathrm{g}} L_{i} / \lambda, \varphi_{\mathrm{s} i}=2 \pi n_{\mathrm{g}} L_{\mathrm{SLR} i} / \lambda, n_{\mathrm{g}}$ is the group index and $\lambda$ is the wavelength.

${ }^{\mathrm{c}} t_{\mathrm{s} i}{ }^{2}+\kappa_{\mathrm{s} i}{ }^{2}=1$ and $t_{\mathrm{b} i}{ }^{2}+\kappa_{\mathrm{b} i}{ }^{2}=1$ for lossless coupling are assumed for all the directional couplers.

\section{ULTRA-SHARP FANO RESONANCES}

In this section, we tailor the spectral response of the zig-zag $3 \mathrm{WC}-\mathrm{SLR}$ resonator to realize optical analogues of Fano resonances with high ERs and SRs. The power transmission spectrum from Port 2 to Port 4 of the zig-zag 3WC-SLR resonator is depicted in Fig. 2(a). The device structural

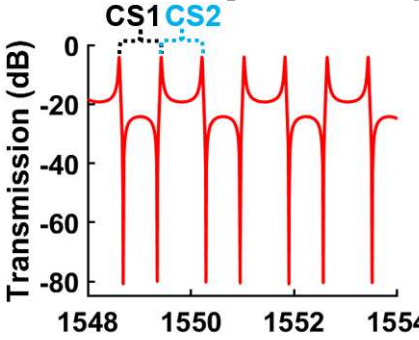

(a)

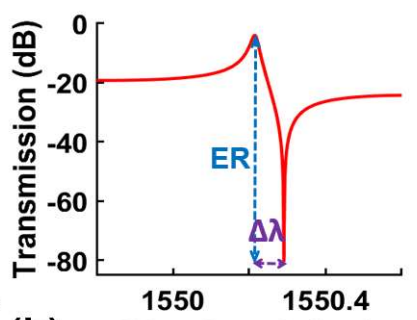

(b) Wavelength $(\mathrm{nm})$
Fig. 2. (a) Power transmission spectrum of the zig-zag $3 \mathrm{WC}-\mathrm{SLR}$ resonator from Port 2 to Port 4 when $L_{\mathrm{SLR}}=L_{1,2,3,4}=115 \mu \mathrm{m}, t_{\mathrm{s}}=0.743$, and $t_{\mathrm{b}}=0.994$. (b) Zoom-in view of (a) in the wavelength range of $1549.8 \mathrm{~nm}-1550.65 \mathrm{~nm}$. CS: channel spacing. ER: extinction ratio. $\Delta \lambda$ : wavelength difference between the resonance peak and notch.

parameters are $L_{\mathrm{SLR}}=L_{1,2,3,4}=115 \mu \mathrm{m}, t_{\mathrm{S}}=0.743$, and $t_{\mathrm{b}}=$ 0.994. One can see that there are periodical Fano resonances with identical asymmetric resonant line-shapes in each period. The FSR is about $200 \mathrm{GHz}$, which equals the sum of the two channel spacings (CS1 and CS2). The two CSs are very close to each other $(\mathrm{CS} 1=101.71 \mathrm{GHz}$ and $\mathrm{CS} 2=98.88 \mathrm{GHz})$, reflecting the high SR of the Fano resonances.

Figure 2(b) shows a zoom-in view of Fig. 2(a) in the wavelength range of $1549.8 \mathrm{~nm}-1550.65 \mathrm{~nm}$, which shows a Fano resonance with an ultra-high ER of $76.32 \mathrm{~dB}$ and an ultra-high SR of $997.66 \mathrm{~dB} / \mathrm{nm}$. The ER is defined as the difference between the maximum and the minimum transmission, and the SR is defined as the ratio of the ER to
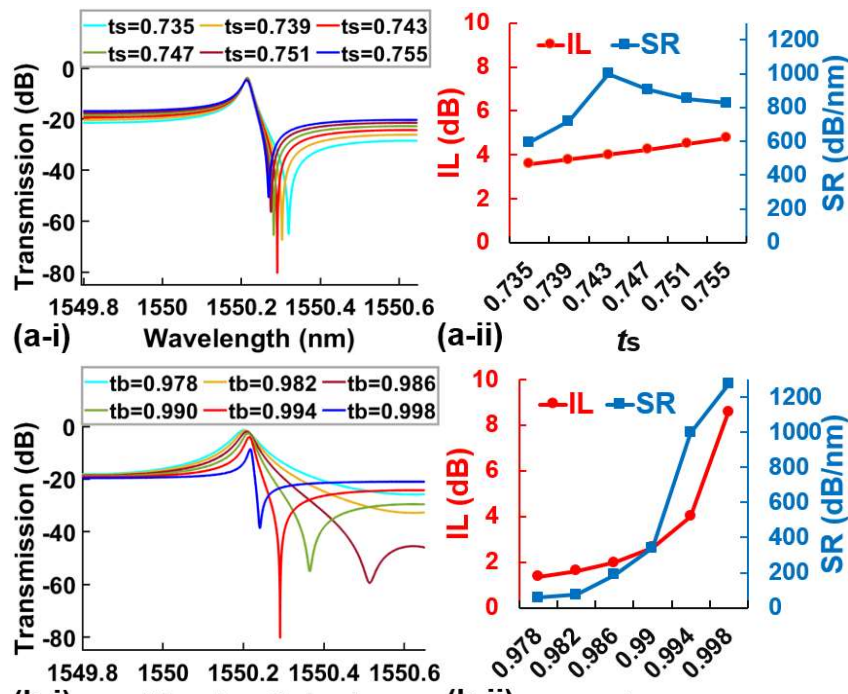

(b-i)

Wavelength $(\mathrm{nm})$
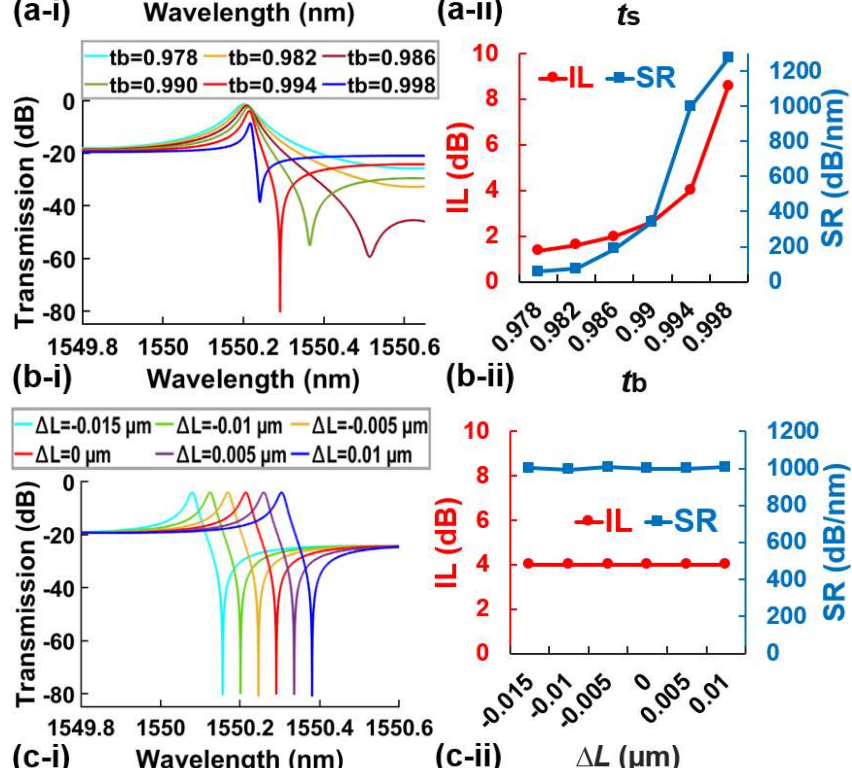

(c-i) Wavelength (nm)

(c-ii) $\quad \Delta L(\mu \mathrm{m})$

Fig. 3. (a-i) Power transmission spectra and (a-ii) the corresponding SR and IL for various $t_{\mathrm{s}}$ when $t_{\mathrm{b}}=0.994$ and $L_{\mathrm{SLR}}=L_{1,2,3,4}=115 \mu \mathrm{m}$, respectively. (b-i) Power transmission spectra and (b-ii) the corresponding IL and SR for various $t_{\mathrm{b}}$ when $t_{\mathrm{s}}=0.743$ and $L_{\mathrm{SLR}}=L=115 \mu \mathrm{m}$, respectively. (c-i) Power transmission spectra and (c-ii) the corresponding IL and SR for different $L=$ $L_{1,2,3,4}$ when $t_{\mathrm{s}}=0.743, t_{\mathrm{b}}=0.994$, and $L_{\mathrm{SLR}}=115 \mu \mathrm{m}$, respectively.

the wavelength difference between the resonance peak and notch (i.e., $\Delta \lambda$ in Fig. 2(b)). The high ER and SR reflect the high performance of the Fano resonances resulting from strong coherent optical mode interference in the compact resonator with only three SLRs. Table II compares the performance of the Fano resonances generated by the parallel 2WC-SLR resonator [164] and the zig-zag 3WC-SLR resonator. As compared with the parallel 2WC-SLR resonator, the zig-zag $3 \mathrm{WC}-\mathrm{SLR}$ resonator can generate Fano resonances with increased ER and SR as well as decreased insertion loss (IL), all of which are highly desirable in practical applications. Moreover, the periodical filter shape of the zig-zag 3WC-SLR resonator is also useful for applications in wavelength division multiplexed (WDM) systems.

TABLE II

PERFORMANCE COMPARISON OF PARALLEL 2WC-SLR AND ZIGZAG 3WC-SLR RESONATORS IN ACHIEVING FANO RESONANCES

\begin{tabular}{c|c|c|c|c|c}
\hline \hline Device & IL & $\begin{array}{c}\text { ER } \\
(\mathbf{d B})\end{array}$ & $\begin{array}{c}\text { SR } \\
(\mathbf{d B})\end{array}$ & $\begin{array}{c}\text { FSR } \\
(\mathbf{d B} / \mathbf{n m})\end{array}$ & (GHz)
\end{tabular}

${ }^{\text {a }}$ The structural parameters are $L_{\mathrm{SLR} i}=L_{i}=115 \mu \mathrm{m}(i=1,2), t_{\mathrm{s}}=0.743$, and $t_{\mathrm{b}}$ $=0.94$. 
In Figs. 3(a) - (c), we further investigate the impact of the device structural parameters including $t_{\mathrm{s}}, t_{\mathrm{b}}$, and $L=L_{1,2,3,4}$ on the performance of the Fano resonances generated by the zigzag 3 WC-SLR resonator. In each figure, we changed only one structural parameter, keeping the others the same as those in Fig. 2. Figure 3(a-i) compares the power transmission spectra for various $t_{\mathrm{s}}$. The corresponding IL and SR are depicted in Fig. 3(a-ii). The IL increases with $t_{\mathrm{s}}$, while the SR first increases and then decreases with $t_{\mathrm{s}}$, achieving a maximum value of $997.66 \mathrm{~dB} / \mathrm{nm}$ at $t_{\mathrm{s}}=0.743$. The non-monotonic relationship between the $\mathrm{SR}$ and $t_{\mathrm{s}}$ is a combined result of both a decrease in $\Delta \lambda$ and a non-monotonic variation in ER. The latter mainly arises from the difference between the internal (transmission) and external (coupling) cavity loss, which is similar to that for different coupling regimes in microring resonators (MRRs) [167]. Figure 3(b-i) compares the power transmission spectra for various $t_{\mathrm{b}}$. The IL and SR functions of $t_{\mathrm{b}}$ are depicted in Fig. 3(b-ii). Both the IL and SR increase with $t_{\mathrm{b}}$, reflecting a trade-off between them. Note that although the ER for $t_{\mathrm{b}}=0.994$ is higher than for $t_{\mathrm{b}}=0.998$, the SR for $t_{\mathrm{b}}$ $=0.994$ is higher than that for $t_{\mathrm{b}}=0.998$ due to a more significantly decreased $\Delta \lambda$. In Figs. $3(\mathrm{c}-\mathrm{i})$ and (c-ii), we compare the corresponding results for various $\Delta L$, which is the length variation of the connecting bus waveguides. To simplify the comparison, we assume the same $\Delta L$ for each connecting bus waveguides $L_{1,2,3,4}$ and keep $L_{\mathrm{SLR}}$ constant. As $\Delta L$ increases, the IL and SR remain unchanged while the resonance redshifts. This highlights the high fabrication tolerance and also indicates that the resonance wavelength can be tuned by introducing thermo-optic micro-heaters $[149,168$, 169] or carrier-injection electrodes [142] along the connecting bus waveguides to tune the phase shift.

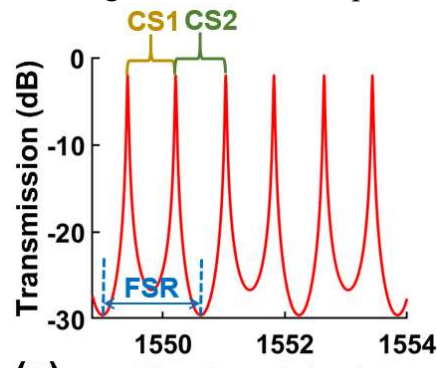

(a)

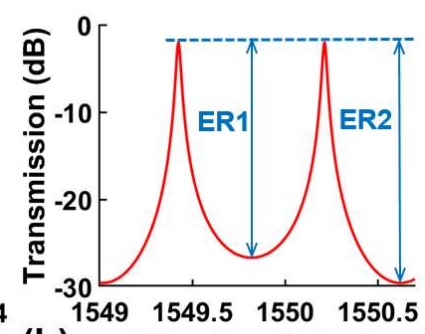

(b)
Wavelength $(\mathbf{n m})$
Fig. 4. (a) Power transmission spectrum of the zig-zag $3 \mathrm{WC}-S L R$ resonator from Port 2 to Port 4 when $L_{\mathrm{SLR}}=L_{1,2,3,4}=115 \mu \mathrm{m}, t_{\mathrm{s}}=0.72$, and $t_{\mathrm{b}}=0.99$. (b) Zoom-in view of (a) in the wavelength range of $1549 \mathrm{~nm}-1550.7 \mathrm{~nm}$.

\section{RESONANCE MODE SPLITTING}

In this section, we tailor the mode interference in the zigzag 3WC-SLR resonator to achieve resonance mode splitting with high ERs and low FSRs. The resonance mode splitting with multiple densely spaced resonances can break the intrinsic dependence between the Q factor, FSR, and physical cavity length, thus allowing low FSRs and high Q factors in resonators with a compact footprint. Figure 4(a) shows the power transmission spectrum from Port 2 to Port 4 of the zigzag $3 \mathrm{WC}-\mathrm{SLR}$ resonator. The structural parameters are $L_{\mathrm{SLR}}=$ $L_{1,2,3,4}=115 \mu \mathrm{m}, t_{\mathrm{s}}=0.72$, and $t_{\mathrm{b}}=0.99$, which are designed in
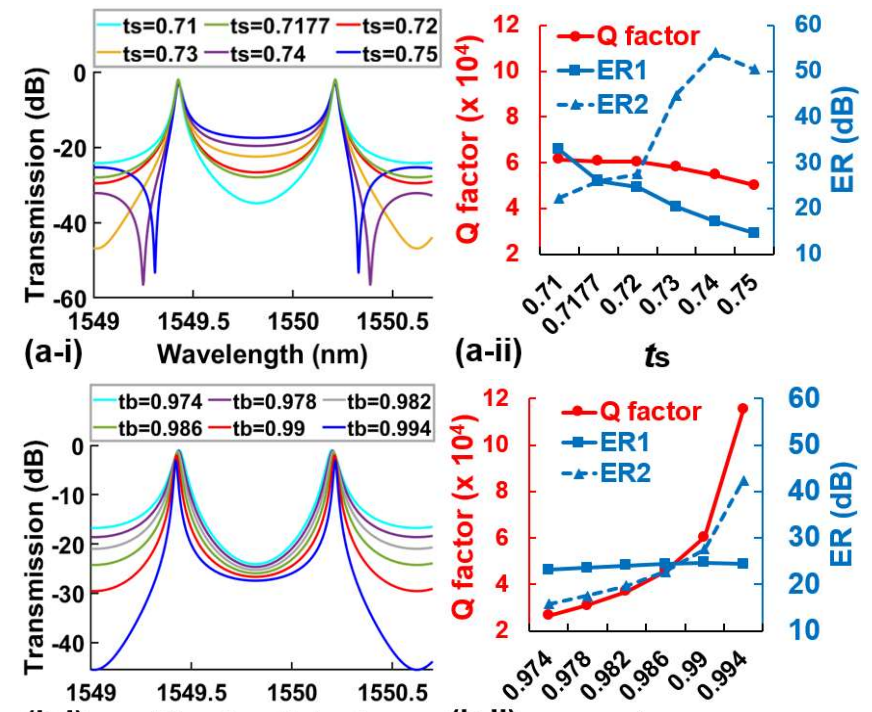

(b-i)

Wavelength $(\mathrm{nm})$
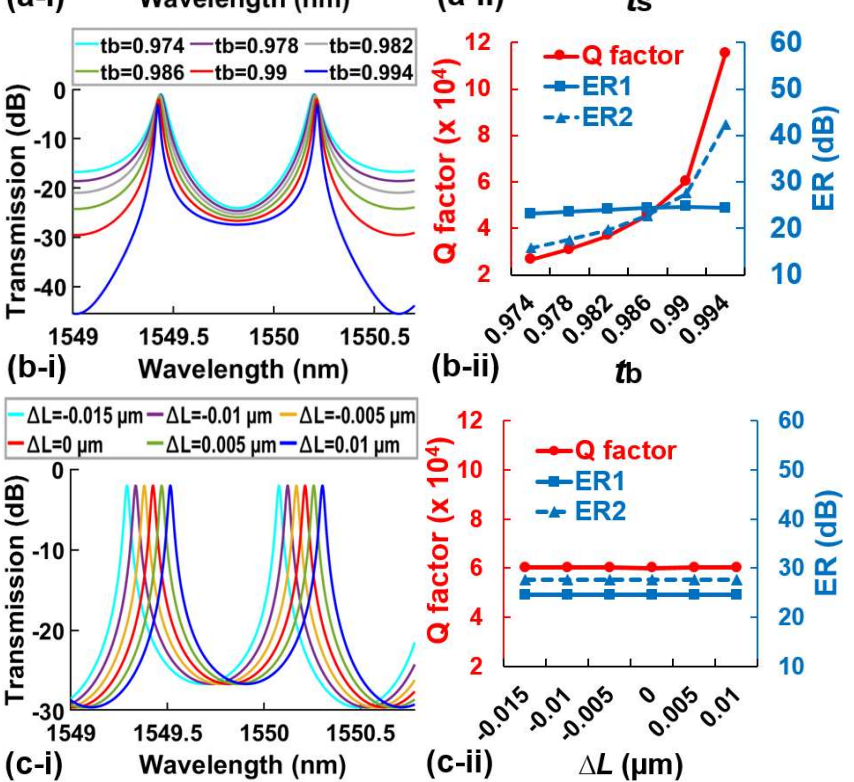

(C-i)

Wavelength (nm)

(c-ii)

$\Delta \boldsymbol{L}(\boldsymbol{\mu m})$

Fig. 5. (a-i) Power transmission spectra of the zig-zag 3WC-SLR resonator for various $t_{\mathrm{s}}$ for input from Port 2 to Port 4 when $t_{\mathrm{b}}=0.99$ and $L_{\mathrm{SLR}}=L_{1,2,3,4}$ $=115 \mu \mathrm{m}$. (a-ii) Calculated Q factor and ERs (ER1 and ER2) as functions of $t_{\mathrm{s}}$ for the transmission spectra in (a-i). (b-i) Power transmission spectra and (b-ii) the corresponding IL and ERs for different $t_{\mathrm{b}}$ when $t_{\mathrm{s}}=0.72$ and $L_{\mathrm{SLR}}=$ $L_{1,2,3,4}=115 \mu \mathrm{m}$, respectively. (c-i) Power transmission spectra and (c-ii) the corresponding Q factor and ERs for different $L$ when $t_{\mathrm{s}}=0.72, t_{\mathrm{b}}=0.99$, and $L_{\mathrm{SLR}}=115 \mu \mathrm{m}$, respectively.

order to achieve a CS of about $100 \mathrm{GHz}$ between adjacent split resonances. In Fig. 4(a), CS1 $=98.33 \mathrm{GHz}$ and CS2 $=102.26$ GHz. There are two split resonances within a FSR of $\sim 200.59$ GHz. Figure 4(b) shows a zoom-in view of Fig. 4(a) in the wavelength range of $1549 \mathrm{~nm}-1550.7 \mathrm{~nm}$. The IL, Q factor, ER1, and ER2 of the two split resonances in Fig. 4(b) are $\sim 2.02 \mathrm{~dB}, \sim 6.03 \times 10^{4}, \sim 24.65 \mathrm{~dB}$, and $\sim 27.55 \mathrm{~dB}$, respectively.

We further investigate the impact of varying $t_{\mathrm{s}}, t_{\mathrm{b}}$, and $L=$ $L_{1,2,3,4}$ on the Q factor, ER1, and ER2 of the split resonances, all of which are important parameters reflecting the degree of mode splitting. In Figs. 5(a) - (c), we only changed one structural parameter in each figure, keeping the others the same as those in Fig. 4. Figure 5(a-i) shows the spectral response for various $t_{\mathrm{s}}$. The Q factor and ERs (ER1 and ER2) as functions of $t_{\mathrm{s}}$ are depicted in Fig. 5(a-ii). As $t_{\mathrm{s}}$ increases, the $\mathrm{Q}$ factor slightly decreases while the ER1 and ER2 change more dramatically, resulting in a change in the spectral response towards that of the Fano resonances in Fig. 2(a). The non-monotonic change in ER2 with $t_{\mathrm{s}}$ follows the trend of the SR in Fig. 3(a-ii) for similar reasons. In particular, ER1 equals to ER2 when $t_{\mathrm{s}}=0.7177$. Under this condition, the $\mathrm{Q}$ factor 


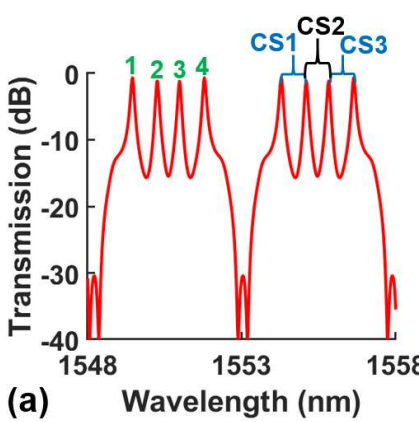

Fig. 6. (a) Power transmission spectrum of the zig-zag $3 \mathrm{WC}-\mathrm{SLR}$ resonator from Port 1 to Port 3 when $L_{\mathrm{SLR}}=115 \mu \mathrm{m}, L_{1,3}=115 \mu \mathrm{m}, L_{2,4}=230 \mu \mathrm{m}$, and $t_{\mathrm{s}}=t_{\mathrm{b}}=0.88$. (b) Zoom-in view of (a) in the wavelength range of $1548.7 \mathrm{~nm}-$ $1550.7 \mathrm{~nm}$

and effective FSR are $\sim 6.06 \times 10^{4}$ and $\sim 100.30 \mathrm{GHz}$ (i.e., half of the FSR in Fig. 4(a)), respectively. To achieve the same FSR, the circumference of a comparable MRR (with the same waveguide geometry and loss) is $690 \mu \mathrm{m}$, which is 6 times the length of the SLRs. This highlights the reduced cavity length enabled by the mode splitting in the $3 \mathrm{WC}-\mathrm{SLR}$ resonator. On the other hand, the $\mathrm{Q}$ factor of a comparable MRR with the same FSR and ER is $\sim 6.08 \times 10^{4}-$ almost the same as that of the zig-zag $3 \mathrm{WC}-\mathrm{SLR}$ resonator. This indicates that the reduced cavity length did not come at the expense of a significant decrease in $\mathrm{Q}$ factor. The spectral response for different $t_{\mathrm{b}}$ are shown in Fig. 5(b-i). The corresponding Q factor and ERs are depicted in Fig. 5(b-ii). The ER1 remains almost unchanged while both the ER2 and the Q factor increase with $t_{\mathrm{b}}$, at the expense of a slightly increased IL. The corresponding results for different $\Delta L$ are shown in Fig. 5(c-i) and (c-ii). Following the trend in Fig. 2(c), the filter shape remains unchanged while the resonance redshifts as $\Delta L$ increases.

The number of split resonances can be changed by varying the length of the connecting bus waveguides. Figure 6(a-i) shows the power transmission spectrum from Port 1 to Port 3 of the zig-zag $3 \mathrm{WC}-\mathrm{SLR}$ resonator. The structural parameters are $L_{\mathrm{SLR}}=115 \mu \mathrm{m}, L_{1,3}=115 \mu \mathrm{m}, L_{2,4}=230 \mu \mathrm{m}$, and $t_{\mathrm{s}}=t_{\mathrm{b}}=$ 0.88 . Clearly, there are four split resonances in each FSR. The CSs between the split resonances are CS1 $=$ CS3 $=100.46$ $\mathrm{GHz}$ and $\mathrm{CS} 2=90.37 \mathrm{GHz}$. Figure 6(b) shows a zoom-in view of Fig. 6(a) in the wavelength range of $1548.7 \mathrm{~nm}-$ $1550.7 \mathrm{~nm}$. To quantitatively analyze the improvement in the performance of the multiple split resonances, in Table III we further compare the resonance mode splitting in the zig-zag $3 \mathrm{WC}-\mathrm{SLR}$ resonator with that of five cascaded SLRs [163] which can also generate four split resonances. As compared with the five cascaded SLRs that only include standing-wave (SW) filter elements, the mode interference between the SW and travelling-wave (TW) filter elements in the zig-zag $3 \mathrm{WC}$ SLR resonator yields higher ERs for the split resonances, a smaller difference between the ERs of the split resonances, and fewer required SLRs.
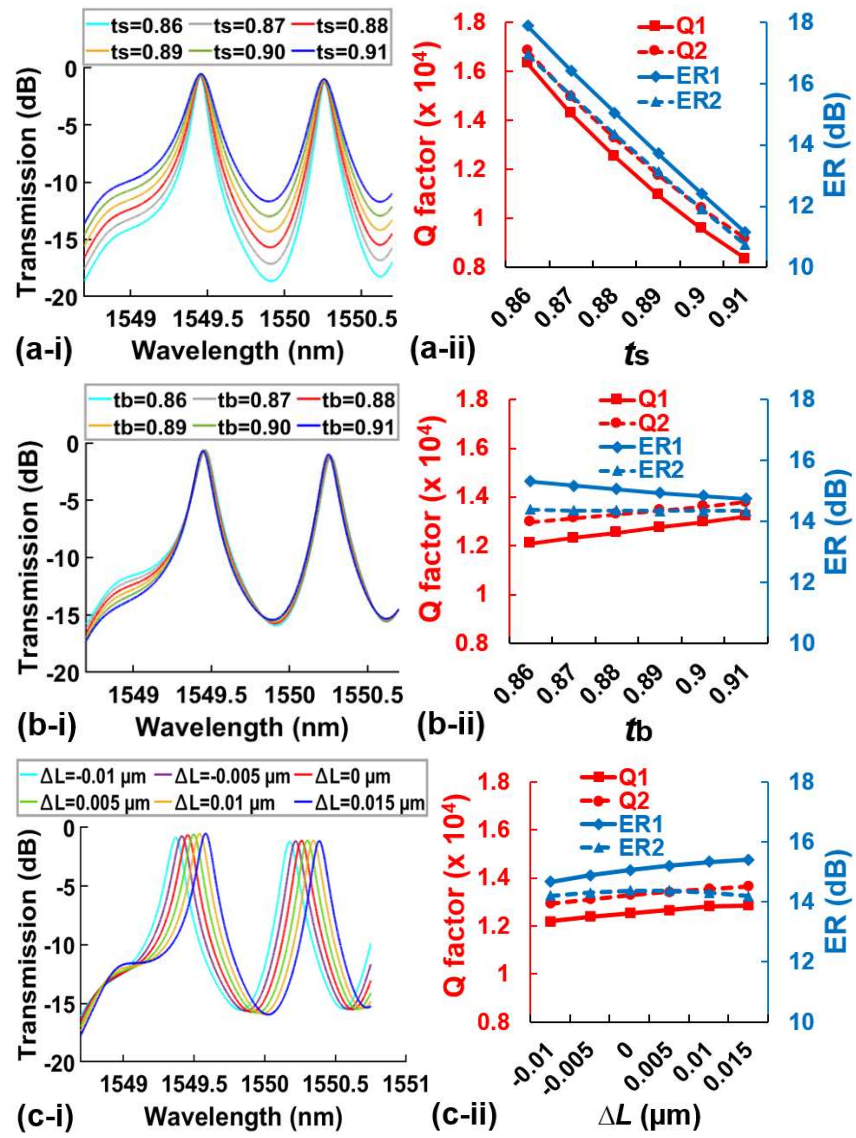

Fig. 7. (a-i) Power transmission spectra of the zig-zag $3 \mathrm{WC}-S L R$ resonator for various $t_{\mathrm{s}}$ for input from Port 1 to Port 3 when $t_{\mathrm{b}}=0.88, L_{\mathrm{SLR}}=115 \mu \mathrm{m}, L_{1,3}=$ $115 \mu \mathrm{m}$, and $L_{2,4}=230 \mu \mathrm{m}$. (a-ii) Calculated Q factors (Q1 and Q2) and ERs (ER1 and ER2) as functions of $t_{\mathrm{s}}$ for the transmission spectra in (a-i). (b-i) Power transmission spectra and (b-ii) the corresponding Q factors and ERs for different $t_{\mathrm{b}}$ when $t_{\mathrm{s}}=0.88, L_{\mathrm{S} L R}=115 \mu \mathrm{m}, L_{1,3}=115 \mu \mathrm{m}$, and $L_{2,4}=230 \mu \mathrm{m}$. (c-i) Power transmission spectra and (c-ii) the corresponding Q factors and ERs versus connecting bus waveguides length variation $\Delta L$ when $L_{\mathrm{SLR}}=115$ $\mu \mathrm{m}$ and $t_{\mathrm{s}}=t_{\mathrm{b}}=0.88$, respectively.

TABLE III

PERFORMANCE COMPARISON OF ZIG-ZAG 3WC-SLR AND FIVE CASCADED SLR RESONATORS IN ACHIEVING MODE SPLITTING

\begin{tabular}{|c|c|c|c|}
\hline & & $\begin{array}{l}\text { Five cascaded } \\
\text { SLR resonator }\end{array}$ & $\begin{array}{l}\text { Zig-zag 3WC-SLR } \\
\text { resonator }\end{array}$ \\
\hline \multicolumn{2}{|c|}{$\begin{array}{c}\text { Number of split } \\
\text { resonances }\end{array}$} & 4 & 4 \\
\hline \multicolumn{2}{|c|}{ Number of SLRs } & 5 & 3 \\
\hline \multirow{2}{*}{ IL (dB) } & IL1, IL4 b & 1.88 & 0.65 \\
\hline & IL2, IL3 & 0.91 & 1.13 \\
\hline \multirow{2}{*}{ ER (dB) } & ER1, ER4 & 5.03 & 15.05 \\
\hline & ER2, ER3 & 4.55 & 14.35 \\
\hline \multirow{3}{*}{ CS (GHz) } & CS1 & 13.8 & 100.46 \\
\hline & CS2 & 16.24 & 90.37 \\
\hline & CS3 & 13.8 & 100.46 \\
\hline \multicolumn{2}{|c|}{ Ref. } & [163] & This work \\
\hline
\end{tabular}

a The structural parameters of the five cascaded SLR resonator are $L_{\mathrm{SLR} i}(i=1-$ $5)=115 \mu \mathrm{m}, L_{i}(i=1-4)=115 \mu \mathrm{m}$, and $t_{\mathrm{si}}(i=1-5)=0.88$.

${ }^{\mathrm{b}}$ ILs of the four split resonances are labelled as IL1-IL4 from left to right. Due to symmetry of the split resonances, there are IL1 = IL4, IL2 = IL3, $\mathrm{ER} 1=\mathrm{ER} 4$, and $\mathrm{ER} 2=\mathrm{ER} 3$. 
TABLE IV

STRUCTURAL PARAMETERS OF CLASSICAL FILTERS BASED ON PARALLEL 3WC-SLR RESONATORS

\begin{tabular}{c|c|c|c|c|c}
\hline \hline & Butterworth & Bessel & Chebyshev Type I & Chebyshev Type II & Elliptic \\
\hline $\boldsymbol{t}_{\mathbf{s}}$ & 0.89 & 0.83 & 0.89 & 0.85 \\
\hline $\boldsymbol{t}_{\mathbf{b}}$ & 0.94 & 0.94 & 0.97 & 0.94 & 0.795 \\
\hline $\boldsymbol{L}_{\mathbf{S L R}}(\boldsymbol{\mu m})$ & 173 & 173 & 173 & 173 & $173(i=1,3)$ \\
\hline $\boldsymbol{L}_{\boldsymbol{i}}(\boldsymbol{\mu m})$ & $173(i=1,3)$ & $173(i=1,3)$ & $173(i=1,3)$ & $346(i=1-4)$ \\
\hline Input $/$ output ports $_{\text {FSR }(\mathbf{G H z})^{\mathbf{a}}}$ & $346(i=2,4)$ & $346(i=2,4)$ & $346(i=2,4)$ & $346(i=2,4)$ \\
\hline \hline
\end{tabular}

${ }^{\mathrm{a}}$ The structural parameters are designed in order to achieve a FSR of $\sim 100.004 \mathrm{GHz}$ in the C band to meet the ITU-T spectral grid standard G694.1 [170].

In Figs. 7(a) - (c), we investigate the impact of $t_{\mathrm{s}}, t_{\mathrm{b}}$, and $\Delta L$ on the performance of the resonance mode splitting based on the zig-zag 3WC-SLR resonator in Fig. 6. We only changed one structural parameter in each figure, keeping the others the same as those in Fig. 6 (a). The power transmission spectra for different $t_{\mathrm{s}}$ and $t_{\mathrm{b}}$ are shown in Figs. 7(a-i) and (b-i), respectively. The corresponding $\mathrm{Q}$ factors $(\mathrm{Q} 1$ and $\mathrm{Q} 2)$ and ERs (ER1 and ER2) for the first two resonances from the left side are shown in Figs. 7(a-ii) and (b-ii) respectively. In Fig. 7(a), all the Q factors and ERs decrease with $t_{\mathrm{s}}$, along with slightly decreased ILs. In Fig. 7(b), as $t_{\mathrm{b}}$ increases, the difference between the two $\mathrm{Q}$ factors as well as the difference between the two ERs gradually decrease, resulting in a more symmetric resonance line-shape, which is desirable for reducing filtering distortions. Figures $7(\mathrm{c}-\mathrm{i})$ and (c-ii) compares the corresponding results for various $\Delta L$. As $\Delta L$ increases, the ER1 slightly increase and ER2 slightly decrease while both $\mathrm{Q}$ factors slightly increase, which make the filter shapes more asymmetric.

\section{HIGH PERFORMANCE CLASSICAL FILTERS}

In this section, we tailor the mode interference in the parallel 3WC-SLR resonator to realize classical filters including Butterworth, Bessel, Chebyshev, and elliptic filters, that all exhibit broad filtering bandwidths and high ERs. The spectral response of these practical filters (solid lines) together with the ideal passband filter (dashed line) are shown in Fig. 8. The Butterworth filter has a flat passband response, while the Bessel filter has a linear phase response over the passband. The Chebyshev filters have either passband ripples (Type I) or stopband ripples (Type II) together with a flat response in the opposite band, resulting in a steeper roll-off than the Butterworth filter. The elliptic filter has both passband and stopband ripples that yields the steepest roll-off among the four types of filters [153].

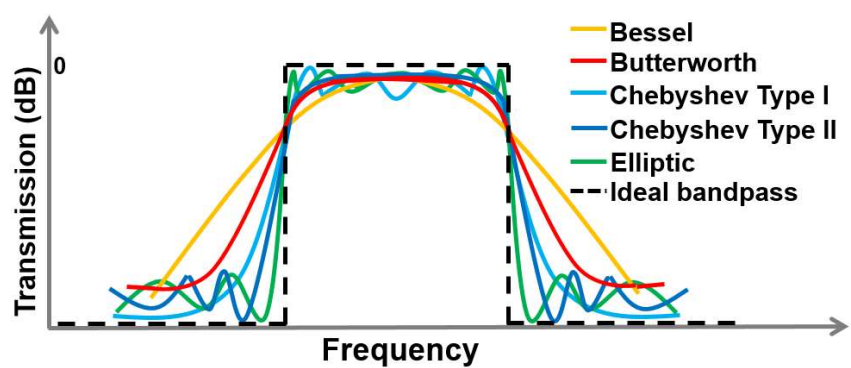

Fig. 8. Spectral responses of classical bandpass filters (solid lines) including Butterworth, Bessel, Chebyshev (Type I and Type II), and elliptic filters. The spectral response of an ideal bandpass filter (dashed lines) is also shown for comparison.

Figure 9(a) shows the power transmission spectrum and corresponding group delay response of the parallel 3WC-SLR resonator from Port 2 to Port 3. As can be seen, there is Butterworth filter shape with flat-top passbands arising from coherent mode interference within the parallel 3WC-SLR resonator, which can be used for low-distortion signal filtering in optical communication systems [171, 172]. The structural parameters are provided in Table IV. The IL, ER, and 3-dB bandwidth (BW) of the Butterworth filter are $\sim 1.71 \mathrm{~dB}, \sim 7.12$ $\mathrm{dB}$, and $\sim 28.08 \mathrm{GHz}$, respectively. We then investigate the impact of $t_{\mathrm{s}}, t_{\mathrm{b}}$, and $\Delta L$ on the performance of the Butterworth filter. The results are shown in Figs. 9(b) - (d), respectively. In Fig. 9(b), the bandwidth of the passband increases with $t_{\mathrm{s}}$, together with slightly degraded filtering flatness. In Fig. 9(c), the resonance is split, with the spectral range between the split resonances increasing with $t_{\mathrm{b}}$. This indicates that the Butterworth filter shape gradually transitions to a Chebyshev Type I filter shape with improved roll-off and degraded flatness. In Fig. 9(d), as $\Delta L$ increases, the filter shape remains unchanged while the resonance redshifts, showing similar trends to the Fano resonances in Fig. 3(c-i) and the resonance mode splitting in Fig. 5(c-i). This reflects the high fabrication tolerance and the feasibility to realize tunable Butterworth filters.

The spectral and group delay responses of the Bessel filter based on the parallel $3 \mathrm{WC}-\mathrm{SLR}$ resonator are shown in Fig. 10(a). The input and output ports are Port 2 and Port 3, respectively, the same as those for the Butterworth filter in Fig. 9. The structural parameters are provided in Table IV. As can be seen, the Bessel filter with a flat-top group delay response is achieved, which is useful for applications such as optical buffering and delay lines $[173,174]$. The group delay response versus $t_{\mathrm{s}}, t_{\mathrm{b}}$, and $\Delta L$ are shown in Figs. 10(b) - (d), 
respectively. In Fig. 10(b), the bandwidth of the group delay response increases with $t_{\mathrm{s}}$, at the expense of a decreased maximum group delay value and degraded flatness. In Fig. $10(\mathrm{c})$, the maximum group delay on both sides increases with $t_{\mathrm{b}}$, while the group delay at the center wavelength shows the opposite trend, resulting in higher unevenness in the group delay response. In Fig. 10(d), the group delay response remains unchanged but redshifts as $\Delta L$ increases.

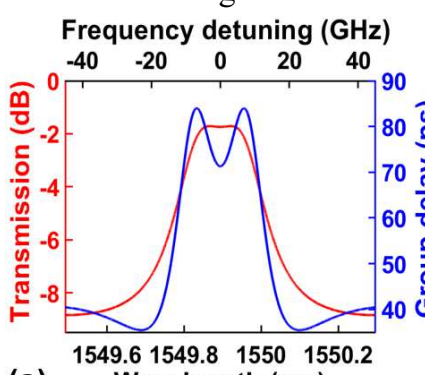

(a)
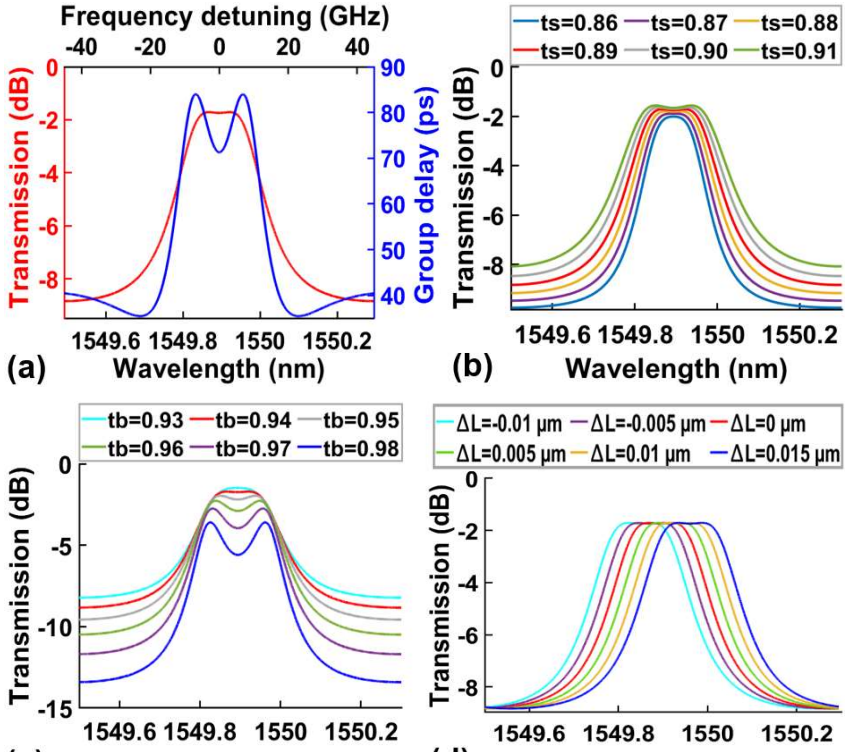

(c) Wavelength $(\mathrm{nm})$

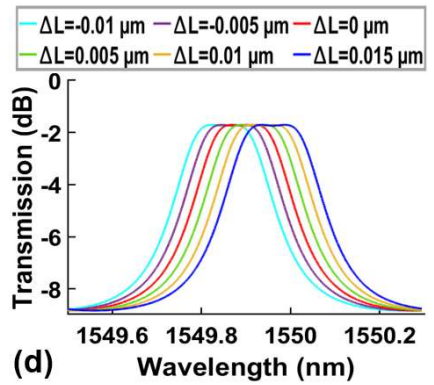

Fig. 9. Butterworth filter based on the parallel 3WC-SLR resonator. (a) Power transmission spectra and group delay response from Port 2 to Port 3. The structural parameters are $L_{\mathrm{SLR}}=173 \mu \mathrm{m}, L_{1,3}=173 \mu \mathrm{m}, L_{2,4}=346 \mu \mathrm{m}, t_{\mathrm{s}}$ $=0.89$, and $t_{\mathrm{b}}=0.94$. (b) $-(\mathrm{d})$ Power transmission spectra versus $t_{\mathrm{s}}, t_{\mathrm{b}}$, and $\Delta L$, respectively. The structural parameters are kept the same as those in (a) except for the varied parameters.

Figure 11(a) shows the power transmission spectrum and group delay response of the Chebyshev Type II filter based on the parallel $3 \mathrm{WC}-\mathrm{SLR}$ resonator. The input port is Port 2, which is the same as those for the Butterworth and Bessel filters, while the output port is changed from Port 3 to Port 4 . The structural parameters are provided in Table IV. Clearly, there is a Chebyshev Type II filter shape with equal stopband ripples and a flat response in the passband. The IL, maximum stopband ripple, ER, and 3-dB BW are $\sim 1.26 \mathrm{~dB}, \sim 0.037 \mathrm{~dB}$, $27.33 \mathrm{~dB}$, and $\sim 18.77 \mathrm{GHz}$, respectively. This filter function with a very flat passband and strongly rejected band can be

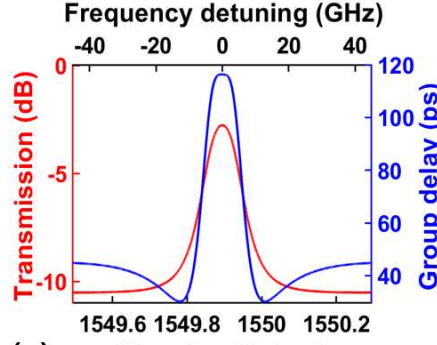

(a)

Wavelength $(\mathrm{nm})$
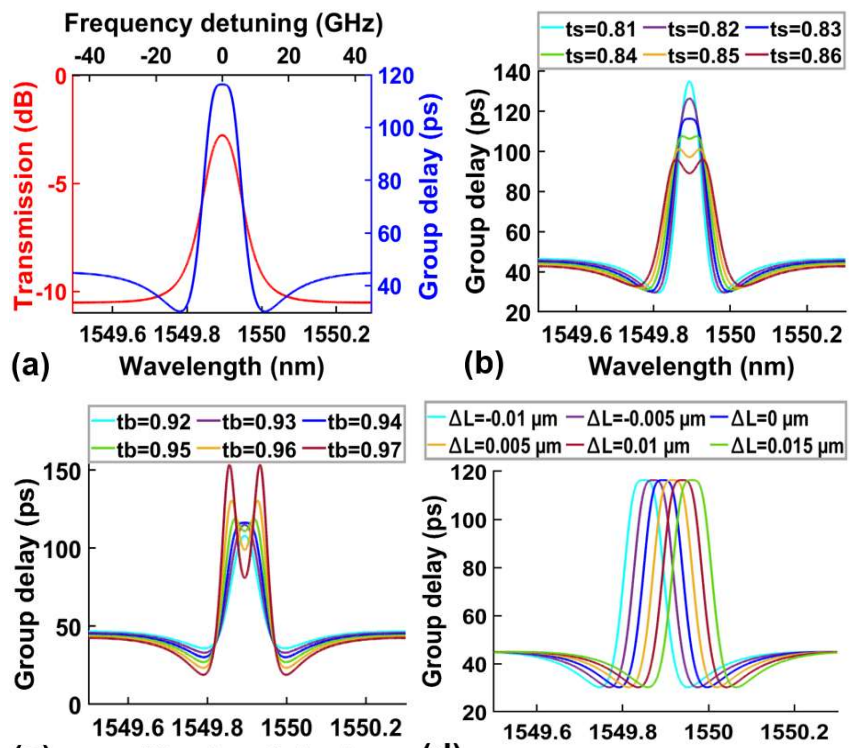

(c) Wavelength (nm)

(d)

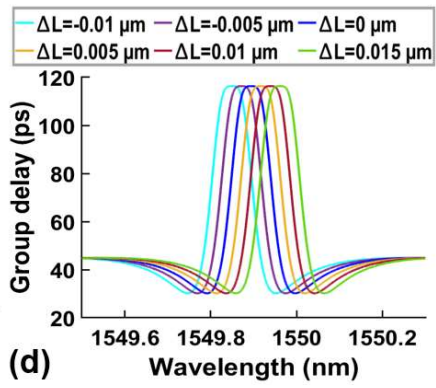

Fig. 10. Bessel filter based on the parallel 3WC-SLR resonator. (a) Power transmission spectra and group delay response from Port 2 to Port 3 when the structural parameters are $L_{\mathrm{SLR}}=173 \mu \mathrm{m}, L_{1,3}=173 \mu \mathrm{m}, L_{2,4}=346 \mu \mathrm{m}, t_{\mathrm{s}}=$ 0.83 , and $t_{\mathrm{b}}=0.94$. (b) - (d) Group delay responses versus $t_{\mathrm{s}}, t_{\mathrm{b}}$, and $\Delta L$, respectively. The structural parameters are kept the same as those in (a) except for the varied parameters.

useful for cleaning and extracting channels from crosstalk in a WDM optical communications system, respectively [175]. The power transmission spectra versus $t_{\mathrm{s}}, t_{\mathrm{b}}$, and $\Delta L$ are shown in Figs. 11(b) - (d), respectively. As shown in Figs. 11(b) and (c), by increasing $t_{\mathrm{s}}$ and keeping constant $t_{\mathrm{b}}$ or vice versa, the notch depth of the single resonance first increases and then the single resonance is split with an increased spectral range between the split resonances. This is a typical phenomenon for resonance mode splitting, which has also been observed in Refs. [149, 176]. In Fig. 11(d), the filter shape remains unchanged but redshifts as $\Delta L$ increases, following the trends for previous filters.

Finally, we tailor the mode interference in the parallel $3 \mathrm{WC}-\mathrm{SLR}$ resonator to realize elliptic filters. As compared with the Butterworth and Chebyshev type I filters that have all-pole transfer functions, elliptic filters include both poles and zeros in the transfer function, which can provide higher stopband extinction levels and better roll-off [160]. Figure 12(a) shows the power transmission spectrum and group delay of the parallel 3WC-SLR resonator from Port 2 to Port 4. There is an elliptic filter shape with ripples in both the passband and stopband. The structural parameters are also provided in Table IV. The IL and notch depth of filter are $\sim 0.59 \mathrm{~dB}$ and $\sim 31.5 \mathrm{~dB}$, respectively. The maximum passband and stopband ripples are $\sim 1.56 \mathrm{~dB}$ and $\sim 18.25 \mathrm{~dB}$, respectively. The ripples in the passband and stopband make the filtering roll-off steeper. These ripples together with the steep roll-off result in a reasonable trade-off between the minimum signal degradation and the maximum noise/interference rejection [177]. The power transmission spectra versus $t_{\mathrm{s}}, t_{\mathrm{b}}$, and $\Delta L$ are shown in Figs. 12(b) $-(\mathrm{d})$, respectively. In Figs. 12(b) and (c), the evolution of the split 

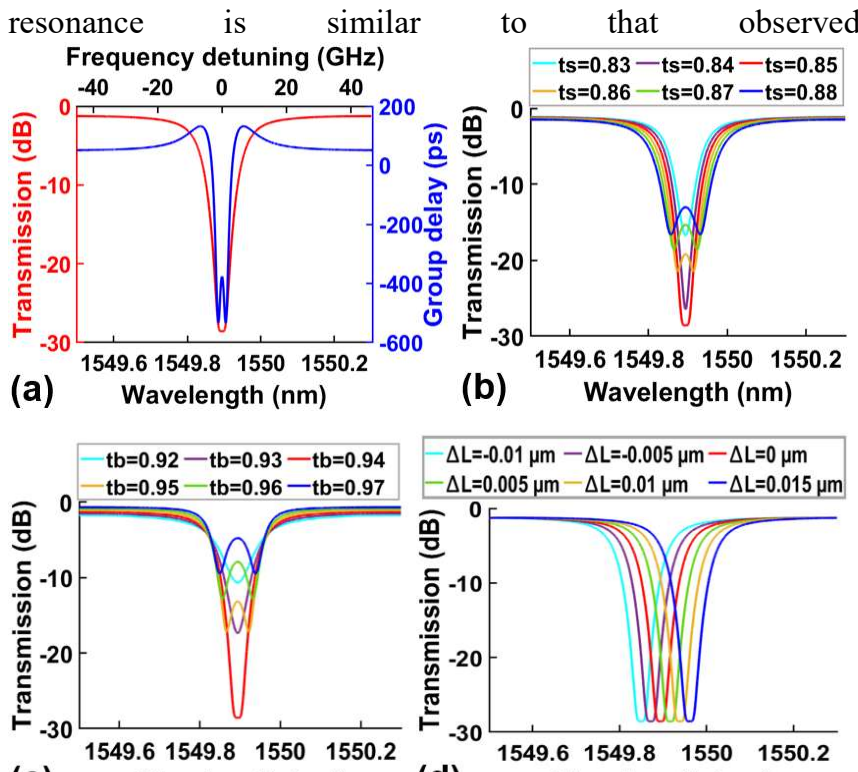

(c) Wavelength $(\mathrm{nm})$

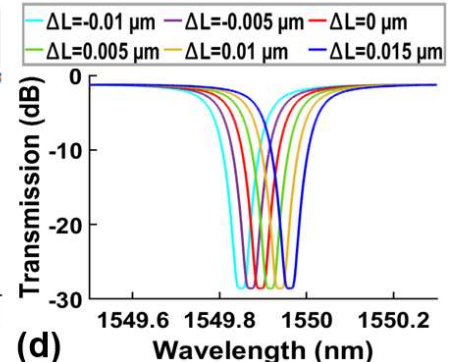

Fig. 11. Chebyshev Type II filter based on the parallel 3WC-SLR resonator (a) Power transmission spectra and corresponding group delay response from Port 2 to Port 4 when $L_{\mathrm{SLR}}=173 \mu \mathrm{m}, L_{1,3}=173 \mu \mathrm{m}, L_{2,4}=346 \mu \mathrm{m}, t_{\mathrm{s}}=0.85$, and $t_{\mathrm{b}}=0.94$. (b) - (d) Power transmission spectra versus $t_{\mathrm{s}}, t_{\mathrm{b}}$, and $\Delta L$, respectively. The structural parameters are kept the same as those in (a) except for the varied parameters.

in Figs. 11 (b) and (c). In Fig. 12(d), unlike the trends for previous filters that exhibit an unchanged filter shape when $\Delta L$ is varied, the filter shape shows a slight asymmetry in the stop-band when $\Delta L$ is away from 0 . This is mainly due to the asynchronous feedback in the elliptic filter, which results in asymmetrically located zeros around the center frequency [178].

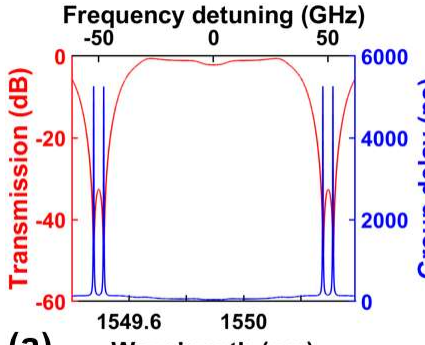

(a)

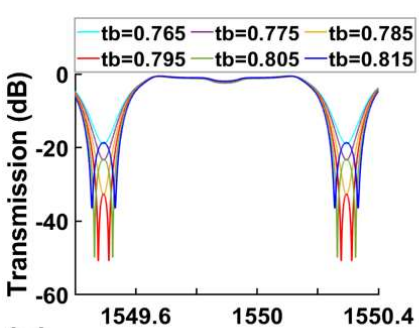

(C) Wavelength $(\mathrm{nm})$

Fig. 12. Elliptic filter based on the parallel 3 WC-SLR resonator. (a) Power transmission spectrum and corresponding group delay response from Port 2 to Port 4. The structural parameters are $L_{\mathrm{SLR}}=692 \mu \mathrm{m}, L_{1,2,3,4}=346 \mu \mathrm{m}, t_{\mathrm{s}}=$ 0.96 , and $t_{\mathrm{b}}=0.795$. (b) - (d) Power transmission spectra versus $t_{\mathrm{s}}, t_{\mathrm{b}}$, and $\Delta L$, respectively. The structural parameters are kept the same as those in (a) except for the varied parameters.

\section{CONCLUSIONS}

We have theoretically investigated advanced multifunctional integrated photonic filters based on 3WC-SLR resonators. Mode interference in the $3 \mathrm{WC}-\mathrm{SLR}$ resonators is tailored to achieve high performance filtering functions including optical analogues of Fano resonances with ultrahigh ERs and SRs, resonance mode splitting with high ERs and low FSRs, and classical Butterworth, Bessel, Chebyshev, and elliptic filters. The requirements for practical applications are considered in our designs, together with detailed analysis of the impact of structural parameters and fabrication tolerances. This work highlights the $3 \mathrm{WC}$-SLR resonators as a powerful and versatile approach to flexible spectral engineering for a diverse range of applications.

\section{REFERENCES}

[1] C. Gunn, "CMOS photonics for high-speed interconnects," IEEE Micro, vol. 26, no. 2, pp. 58-66, Mar.-Apr. 2006.

[2] W. Bogaerts and L. Chrostowski, "Silicon photonics circuit design: methods, tools and challenges," Laser Photon. Rev., vol. 12, no. 4, pp. 1700237 (1-29), Apr. 2018.

[3] W. Bogaerts et al., "Silicon microring resonators," Laser Photon. Rev., vol. 6, no. 1, pp. 47-73, Jan. 2012.

[4] H. Arianfard and R. Ghayour, "Nanoscale plasmonic filter based on coupled metal-insulator-metal waveguides using nonlinear nanoslot resonator," J. Nanophoton., vol. 9, no. 1, Dec. 2014, Art. no. 093799.

[5] S. Feng et al., "Silicon photonics: from a microresonator perspective," Laser Photon. Rev., vol. 6, no. 2, pp. 145-177, Apr. 2012.

[6] Z. Yao et al., "Integrated silicon photonic microresonators: emerging technologies," IEEE J. Sel. Top. Quantum Electron., vol. 24, no. 6, pp. 1-24, Nov.-Dec. 2018.

[7] J. Wu et al., "2D layered graphene oxide films integrated with micro-ring resonators for enhanced nonlinear optics," Small, vol. 16, no. 16, pp. 1906563 (1-12), Apr. 2020

[8] J. Wu et al., "Compact tunable silicon photonic differentialequation solver for general linear time-invariant systems," Opt. Express, vol. 22, no. 21, pp. 26254-64, Oct. 2014.

[9] H. Arianfard, B. Khajeheian, and R. Ghayour, "Tunable band (pass and stop) filters based on plasmonic structures using Kerr-type nonlinear rectangular nanocavity," Opt. Eng., vol. 56, no. 12 , Dec. 2016, Art. no. 121902.

[10] W. Liu et al., "A fully reconfigurable photonic integrated signal processor,” Nat. Photon., vol. 10, no. 3, pp. 190-195, Feb. 2016.

[11] D. J. Moss, M. Lamont, S. Mclaughlin, G. Randall, P. Colbourne, S. Kiran and C. A. Hulse, "Tunable dispersion and dispersion slope compensators for $10 \mathrm{~Gb} / \mathrm{s}$ using all-pass multicavity etalons", IEEE Photonics Technology Letters, vol. 15, no. 5, 730-732 (2003). DOI: 10.1109/LPT.2003.809921.

[12] L.M. Lunardi et al., "Tuneable dispersion compensators based on multi-cavity all-pass etalons for $40 \mathrm{~Gb} / \mathrm{s}$ systems", Journal of Lightwave Technology, vol. 20, no. 12, 2136 (2002). DOI: 10.1109/JLT.2002.806768.

[13] D. J. Moss et al., "Multichannel tunable dispersion compensation using all-pass multicavity etalons", paper TuT2 Optical Fiber Communications Conference, Anaheim (2002). Post-conference Technical Digest (IEEE Cat. No.02CH37339). Opt Soc. America. Part vol.1, 2002, pp. 132-3. Washington, DC, USA.

[14] L.M. Lunardi, D. Moss, S. Chandrasekhar, L.L. Buhl, "An etalon based tunable dispersion compensator (TDC) device for $40 \mathrm{~Gb} / \mathrm{s}$ applications", European Conference on Optical Communications (ECOC), paper 5.4.6, Copenhagen, Sept. (2002). IEEE. Part vol. 2, 2002, pp. 2 vol. 2. Piscataway, NJ, USA. INSPEC Accession Number: 9153476, Print ISBN: 87-90974-63-8.

[15] D.J. Moss, L.M. Lunardi, MRE Lamont, G. Randall, P. Colbourne, S. Chandrasekhar, L. Buhl, "Tunable dispersion compensation at 10 $\mathrm{Gb} / \mathrm{s}$ and $40 \mathrm{~Gb} / \mathrm{s}$ using multicavity all-pass etalons", Optical Fiber 
Communications Conference, paper TuD1, page 162, Atlanta, GA March (2003) (Trends in Optics and Photonics Series Vol.86) Postconference Digest (IEEE Cat. No.03CH37403). Opt. Soc. America. Part vol.1, 2003, pp. 162-3. Washington, DC, USA.

[16] J. S. Levy, A. Gondarenko, M. A. Foster, et al., "CMOS-compatible multiple-wavelength oscillator for on-chip optical interconnects," Nature Photonics, vol. 4, 1, pp. 37-40, 2010.

[17] L. Razzari, D. Duchesne, M. Ferrera, et al., "CMOS-compatible integrated optical hyper-parametric oscillator," Nature Photonics, vol. 4 , no. 1 , pp. $41-45,2010$

[18] D. J. Moss, R. Morandotti, A. L. Gaeta, et al., "New CMOScompatible platforms based on silicon nitride and Hydex for nonlinear optics," Nature Photonics, vol. 7, no. 8, pp. 597-607, 2013.

[19] A. Pasquazi et al., "Micro-combs: A novel generation of optical sources," Physics Reports, vol. 729, pp. 1-81, Jan 27. 2018.

[20] P. Del'Haye, A. Schliesser, O. Arcizet, T. Wilken, R. Holzwarth, and T. J. Kippenberg, "Optical frequency comb generation from a monolithic microresonator", Nature, vol. 450, pp. 1214-1217, 2007.

[21] M. Peccianti, et al., "Demonstration of an ultrafast nonlinear microcavity modelocked laser", Nature Communications, vol. 3, pp. 765, 2012. DOI:10.1038/ncomms 1762

[22] M. Kues, et al., "Passively modelocked laser with an ultra-narrow spectral width", Nature Photonics, vol. 11, no. 3, pp. 159, 2017. DOI:10.1038/nphoton.2016.271

[23] A. Pasquazi, L. Caspani, M. Peccianti, et al., "Self-locked optical parametric oscillation in a CMOS compatible microring resonator: a route to robust optical frequency comb generation on a chip," Optics Express, vol. 21, no. 11,pp. 13333-13341, 2013

[24] A. Pasquazi, M. Peccianti, B. E. Little, et al., "Stable, dual mode, high repetition rate mode-locked laser based on a microring resonator," Optics Express, vol. 20, no. 24, pp. 27355-27362, 2012.

[25] H. Bao, et al., "Laser cavity-soliton microcombs," Nature Photonics, vol. 13, no. 6, pp. 384-389, Jun. 2019.

[26] X. Xue, X. Zheng, and B. Zhou, "Super-efficient temporal solitons in mutually coupled optical cavities," Nature Photonics, May 2019.

[27] H. Zhou, et al., "Soliton bursts and deterministic dissipative Kerr soliton generation in auxiliary-assisted microcavities," Light: Science \& Applications, vol. 8, no. 1, pp. 50, 2019.

[28] H. Bao et al., "Turing patterns in a fibre laser with a nested microresonator: robust and controllable micro-comb generation", Physical Review Research, vol. 2, pp. 023395, 2020.

[29] L. D. Lauro, J. Li, D. J. Moss, R. Morandotti, S. T. Chu, M. Peccianti, and A. Pasquazi, "Parametric control of thermal selfpulsation in micro-cavities," Opt. Lett. vol. 42, no. 17, pp. $3407-$ 3410, Aug. 2017.

[30] H. Bao, et al., "Type-II micro-comb generation in a filter-driven four wave mixing laser," Photonics Research, vol. 6, no. 5, pp. B67-B73, May 2018.

[31] B. Shen, Chang, L., Liu, J., et al., "Integrated turnkey soliton microcombs," Nature, vol. 582, pp. 365-369, 2020.

[32] T. G. Nguyen et al., "Integrated frequency comb source-based Hilbert transformer for wideband microwave photonic phase analysis," Opt. Express, vol. 23, no. 17, pp. 22087-22097, Aug. 2015.

[33] J. Wu, X. Xu, T. G. Nguyen, S. T. Chu, B. E. Little, R. Morandotti, A. Mitchell, and D. J. Moss, "RF Photonics: An Optical Microcombs' Perspective," IEEE J. Sel. Top. Quantum Electron., vol. 24, no. 4, pp. 6101020, Jul-Aug. 2018. DOI: 10.1109/JSTQE.2018.2805814.

[34] X. Xue, et al., "Programmable single-bandpass photonic RF filter based on a Kerr comb from a microring," Journal of Lightwave Technol., vol. 32, no. 20, pp. 3557-3565, Oct. 2014.

[35] X. Xu et al., "Reconfigurable broadband microwave photonic intensity differentiator based on an integrated optical frequency comb source," APL Photonics, vol. 2, no. 9, 096104, Sep. 2017.

[36] X. Xu, M. Tan, J. Wu, R. Morandotti, A. Mitchell, and D. J. Moss, "Microcomb-based photonic RF signal processing", IEEE Photonics Technology Letters, vol. 31 no. 23 1854-1857, 2019.

[37] X. Xu, et al., "Advanced RF and microwave functions based on an integrated optical frequency comb source," Opt. Express, vol. 26, no. 3, pp. 2569-2583, Feb. 2018.

[38] X. Xue, et al., "Microcomb-based true-time-delay network for microwave beamforming with arbitrary beam pattern control," Journal of Lightwave Technology, vol. 36, no. 12, pp. 2312-2321, Jun. 2018.
[39] X. Xu, et al., "Broadband RF channelizer based on an integrated optical frequency Kerr comb source," Journal of Lightwave Technology, vol. 36, no. 19, pp. 4519-4526, 2018.

[40] X. Xu, et al., "Continuously tunable orthogonally polarized RF optical single sideband generator based on micro-ring resonators," Journal of Optics, vol. 20, no. 11, 115701. 2018.

[41] X. Xu, et al., "Orthogonally polarized RF optical single sideband generation and dual-channel equalization based on an integrated microring resonator," Journal of Lightwave Technology, vol. 36, no. 20, pp. 4808-4818. 2018.

[42] X. Xu, et al., "Photonic microwave true time delays for phased array antennas using a $49 \mathrm{GHz}$ FSR integrated optical micro-comb source," Photonics Research, vol. 6, no. 5, pp. B30-B36, 2018.

[43] X. Xu, et al., "Advanced adaptive photonic RF filters with 80 taps based on an integrated optical micro-comb source," Journal of Lightwave Technology, vol. 37, no. 4, pp. 1288-1295, 2019.

[44] W. Liang, et al., "High spectral purity Kerr frequency comb radio frequency photonic oscillator," Nature Communications, vol. 6 pp. 7957. 2015.

[45] J. Liu, et al., "Photonic microwave generation in the X-and K-band using integrated soliton microcombs" Nature Photonics, vol. 14, pp. $1-6,2020$.

[46] X. Xu, et al., Broadband microwave frequency conversion based on an integrated optical micro-comb source", Journal of Lightwave Technology, vol. 38 no. 2, pp. 332-338, 2020.

[47] X. Xu, et al., "Broadband photonic RF channelizer with 90 channels based on a soliton crystal microcomb", Journal of Lightwave Technology, Vol. 38, no. 18, pp. 5116 - 5121, 2020. doi: 10.1109/JLT.2020.2997699.

[48] X. Xu, et al., "Photonic RF and microwave integrator with soliton crystal microcombs", IEEE Transactions on Circuits and Systems II: Express Briefs, Vol. 67, No. 12, pp. 3582-3586 (2020). DOI:10.1109/TCSII.2020.2995682.

[49] X. Xu, et al., "Photonic RF phase-encoded signal generation with a microcomb source", Journal of Lightwave Technology, vol. 38, no. 7, pp. 1722-1727, 2020.

[50] X. Xu, et al., "High performance RF filters via bandwidth scaling with Kerr micro-combs," APL Photonics, vol. 4, no. 2, pp. 026102. 2019.

[51] M. Tan, et al., "Microwave and RF photonic fractional Hilbert transformer based on a $50 \mathrm{GHz}$ Kerr micro-comb", Journal of Lightwave Technology, vol. 37, no. 24, pp. 6097 - 6104, 2019.

[52] M. Tan, et al., "RF and microwave fractional differentiator based on photonics", IEEE Transactions on Circuits and Systems: Express Briefs, Vol. 67, No. 11, pp. 2767-2771 (2020). DOI:10.1109/TCSII.2020.2965158.

[53] M. Tan, et al., "Photonic RF arbitrary waveform generator based on a soliton crystal micro-comb source", Journal of Lightwave Technology, vol. 38, no. 22, pp. 6221 - 6226, Oct 22 (2020).

[54] M. Tan, X. Xu, J. Wu, R. Morandotti, A. Mitchell, and D. J. Moss, "RF and microwave high bandwidth signal processing based on Kerr Micro-combs", Advances in Physics X, vol. 6, no. 1, 1838946 (2021).

[55] M. Tan, X. Xu, J. Wu, R. Morandotti, A. Mitchell, and D. J. Moss, "Photonic RF and microwave filters based on $49 \mathrm{GHz}$ and $200 \mathrm{GHz}$ Kerr microcombs", Optics Communications, vol. 465, Article: 125563 (2020)

[56] B. Corcoran, et al., "Ultra-dense optical data transmission over standard fiber with a single chip source", Nature Communications, vol. 11, Article:2568, 2020. DOI:10.1038/s41467-020-16265-x.

[57] P. Marin-Palomo, et al., "Microresonator-based solitons for massively parallel coherent optical communications", Nature, vol. 546, no. 7657, pp. 274, 2017.

[58] J. Pfeifle, V. Brasch, M. Lauermann, Y. Yu, D. Wegner, T. Herr, K. Hartinger, et al., "Coherent terabit communications with microresonator Kerr frequency combs", Nature Photonics, vol. 8, no. 5, pp. 375-380, 2014.

[59] X. Xu, et al., "Photonic perceptron based on a Kerr microcomb for scalable high speed optical neural networks", Laser and Photonics Reviews, vol. 14, no. 8, 2000070, 2020. DOI:10.1002/lpor.202000070.

[60] X. Xu, et al., "11 TeraFLOPs per second photonic convolutional accelerator for deep learning optical neural networks", $\mathbf{5 8 9}$ (7840) 44-51 (2021) 
[61] C. Reimer, L. Caspani, M. Clerici, et al., "Integrated frequency comb source of heralded single photons," Optics Express, vol. 22, no. 6 , pp. $6535-6546,2014$

[62] C. Reimer, et al., "Cross-polarized photon-pair generation and bichromatically pumped optical parametric oscillation on a chip", Nature Communications, vol. 6, Article 8236, 2015. DOI: $10.1038 /$ ncomms 9236

[63] L. Caspani, C. Reimer, M. Kues, et al., "Multifrequency sources of quantum correlated photon pairs on-chip: a path toward integrated Quantum Frequency Combs," Nanophotonics, vol. 5, no. 2, pp. 351$362,2016$.

[64] C. Reimer, M. Kues, P. Roztocki, B. Wetzel, F. Grazioso, B. E. Little, S. T. Chu, T. Johnston, Y. Bromberg, L. Caspani, D. J. Moss, and R. Morandotti, "Generation of multiphoton entangled quantum states by means of integrated frequency combs," Science, vol. 351, no. 6278, pp. 1176-1180, 2016.

[65] M. Kues, et al., "On-chip generation of high-dimensional entangled quantum states and their coherent control", Nature, vol. 546, no. 7660, pp. 622-626, 2017.

[66] P. Roztocki, M. Kues, C. Reimer, B. Wetzel, S. Sciara, Y. Zhang, A Cino, B. E. Little, S. T. Chu, D. J. Moss, and R. Morandotti, "Practical system for the generation of pulsed quantum frequency combs," Optics Express, vol. 25, no. 16, pp. 18940-18949, 2017.

[67] Y. Zhang, et al., "Induced photon correlations through superposition of two four-wave mixing processes in integrated cavities", Laser and Photonics Reviews, vol. 14, no. 7, pp. 2000128, 2020. DOI: 10.1002/lpor.202000128

[68] M. Kues, C. Reimer, A. Weiner, J. Lukens, W. Munro, D. J. Moss, and R. Morandotti, "Quantum Optical Micro-combs", Nature Photonics, vol. 13, no.3, pp. 170-179, 2019.

[69] C. Reimer, et al.,"High-dimensional one-way quantum processing implemented on d-level cluster states", Nature Physics, vol. 15, no.2, pp. 148-153, 2019

[70] M. Lee, et al., "Photosensitive post tuning of chalcogenide photonic crystal waveguides," Optics Express, vol. 15, no. 3, pp. 1277-1285, 2007. DOI:10.1364/OE.15.001277

[71] S. Tomljenovic-Hanic, M. J. Steel, C. M. d. Sterke and D. J. Moss, "High-Q cavities in photosensitive photonic crystals", Optics Letters, 32, no. 5, pp. 542-544, 2007.

[72] C. Grillet, et al., "Nanowire coupling to photonic crystal nanocavities for single photon sources," Optics Express, vol. 15, no. 3, pp. 1267-1276, 2007. DOI:10.1364/OE.15.001267

[73] D. Freeman, et al., "Chalcogenide Glass Photonic Crystal Devices", Photonic and Electromagnetic Crystal Structures, Photonics and Nanostructures-Fundamentals and Applications, Science Direct Elsevier Publishing, vol. 6, no. 1, pp. 3-11, 2008 doi:10.1016/j.photonics.2007.11.001.

[74] C. Grillet, et al., "Characterization and modeling of Fano resonances in chalcogenide photonic crystal membranes", Optics Express, vol. 14 , no. 1 , pp. 369-376, 2006.

[75] M. Lee, et al., "Photosensitive post tuning of chalcogenide photonic crystal waveguides," Optics Express, vol. 15, no. 3, pp. 1277-1285, 2007. DOI:10.1364/OE.15.001277

[76] S. Tomljenovic-Hanic, M. J. Steel, C. M. d. Sterke and D. J. Moss, "High-Q cavities in photosensitive photonic crystals", Optics Letters, 32, no. 5, pp. 542-544, 2007.

[77] M. Ferrera, et al., "All-optical $1^{\text {st }}$ and $2^{\text {nd }}$ order integration on a chip,” Optics Express, vol. 19, no. 23, pp. 23153-23161, 2011.

[78] M. Ferrera, L. Razzari, D. Duchesne, et al., "Low-power continuouswave nonlinear optics in doped silica glass integrated waveguide structures," Nature Photonics, vol. 2, no. 12, pp. 737-740, 2008.

[79] M. Ferrera, D. Duchesne, L. Razzari, et al., "Low power four wave mixing in an integrated, micro-ring resonator with $\mathrm{Q}=1.2$ million," Optics Express, vol. 17, no. 16, pp. 14098-14103, 2009.

[80] D. Duchesne, M. Peccianti, M. R. E. Lamont, et al., "Supercontinuum generation in a high index doped silica glass spiral waveguide," Optics Express, vol. 18, no, 2, pp. 923-930, 2010.

[81] M. Ferrera, et al., "On-chip CMOS-compatible all-optical integrator", Nature Communications, vol. 1, Article 29, 2010. DOI:10.1038/ncomms 1028
[82] A. Pasquazi, et al., "All-optical wavelength conversion in an integrated ring resonator," Optics Express, vol. 18, no. 4, pp. 38583863, 2010.

[83] A. Pasquazi, Y. Park, J. Azana, et al., "Efficient wavelength conversion and net parametric gain via Four Wave Mixing in a high index doped silica waveguide," Optics Express, vol. 18, no. 8, pp. 7634-7641, 2010.

[84] B. Wetzel et al., "Smart supercontinuum sources: Customizing nonlinear optical interactions via adaptive temporal pulse-splitting", Nature Communications, Vol. 9, Article 4884, November 20 (2018).

[85] M. Peccianti, M. Ferrera, L. Razzari, et al., "Sub-picosecond optical pulse compression via an integrated nonlinear chirper," Optics Express, vol. 18, no. 8, pp. 7625-7633, 2010.

[86] D. Duchesne, M. Ferrera, L. Razzari, et al., "Efficient self-phase modulation in low loss, high index doped silica glass integrated waveguides," Optics Express, vol. 17, no. 3, pp. 1865-1870, 2009.

[87] M. Ferrera, et al., "CMOS compatible integrated all-optical radio frequency spectrum analyzer," Optics Express, vol. 22, no. 18, pp. 21488 - 21498, 2014. DOI: 10.1364/OE.22.021488.

[88] L. Jin, L. Di Lauro, A. Pasquazi, M. Peccianti, D. J. Moss, R. Morandotti, B.E. Little, S.T. Chu, "Optical multi-stability in a nonlinear high-order microring resonator filter", Applied Physics Letters (APL) Photonics, Vol. 5, Article 056106, May 22 (2020).

[89] M. Rochette, et al., "Bit-error-ratio improvement with 2R optical regenerators," IEEE Photonics Technology Letters, vol. 17, no. 4, pp. 908-910, 2005.

[90] F. Li, et al., "All-optical XOR logic gate for 40Gb/s DPSK signals via FWM in a silicon nanowire," Optics Express, vol. 19, no. 21, pp. 20364-20371, 2011. DOI: 10.1364/OE.19.020364.

[91] F. Li, et al., "Error-free All-Optical Demultiplexing at $160 \mathrm{~Gb} / \mathrm{s}$ via FWM in a Silicon Nanowire," Optics Express, vol. 18, no. 4, pp. 3905-3910, 2010. DOI: 10.1364/OE.18.003905.

[92] H. Ji, et al., "1.28-Tb/s Demultiplexing of an OTDM DPSK Data Signal Using a Silicon Waveguide," Photonics Technology Letters, vol. 22, no. 23, pp. 1762-1764, 2010.

[93] C. Monat, et al., "Investigation of phase matching for third-harmonic generation in silicon slow light photonic crystal waveguides using Fourier optics," Optics Express, vol. 18, no. 7, pp. 6831-6840, 2010. DOI: 10.1364/OE.18.006831.

[94] B. Corcoran, et al., "Optical Signal Processing on a Silicon Chip at 640Gb/s Using Slow-Light," Optics Express, vol. 18, no. 8, pp. 7770-7781, 2010. DOI: 10.1364/OE.18.007770.

[95] B. Corcoran, et al., "Silicon nanowire based radio-frequency spectrum analyzer," Optics Express, vol. 18, no. 19, pp. 2019020200, 2010. DOI: 10.1364/OE.18.020190.

[96] C. Monat, et al., "Integrated optical auto-correlator based on thirdharmonic generation in a silicon photonic crystal waveguide," Nature Communications, vol. 5, Article 3246, 2014. doi: $10.1038 /$ ncomms 4246

[97] D. J. Moss, H. M. van Driel, and J. E. Sipe, "Dispersion in the anisotropy of optical third-harmonic generation in silicon," Opt. Lett., vol. 14, no. 1, pp. 57-59, 1989.

[98] J. E. Sipe, D. J. Moss, and H. M. van Driel, "Phenomenological Theory of Optical Second- And Third-Harmonic Generation Form Cubic Centrosymmetric Crystals," Phys. Rev. B, vol. 35, no. 3, pp. 1129-1141, 1987.

[99] T. D. Vo, et al., "Silicon-Chip-Based Real-Time Dispersion Monitoring for 640 Gbit/s DPSK Signals," IEEE Journal of Lightwave Technology, vol. 29, no. 12, pp. 1790-1796, 2011.

[100] F. Li, et al., "All-optical wavelength conversion for $10 \mathrm{~Gb} / \mathrm{s}$ DPSK signals in a silicon ring resonator," Optics Express, vol. 19, no. 23, pp. 22410-22416, 2011.

[101] B. Corcoran, et al., "Green light emission in silicon through slowlight enhanced third-harmonic generation in photonic-crystal waveguides," Nature Photonics, vol. 3, no. 4, pp. 206-210, 2009. doi:10.1038/nphoton.2009.28.

[102] D. J. Moss, E. Ghahramani, J. E. Sipe, and H. M. van Driel, "Bandstructure calculation of dispersion and anisotropy in $\chi \rightarrow(3)$ for third- 
harmonic generation in Si, Ge, and GaAs," Phys. Rev. B, vol. 41, no. 3, pp. 1542-1560, 1990.

[103] D. J. Moss, H. M. van Driel, and J. E. Sipe, "Third harmonic generation as a structural diagnostic of ion implanted amorphous and crystalline silicon," Appl. Phy. Lett., vol. 48, no. 17, pp. 1150, 1986.

[104] M.D. Pelusi, F. Luan, E. Magi, M.R.E. Lamont, D. J. Moss, B.J. Eggleton, J.S. Sanghera, L. B. Shaw, and I.D. Aggarwal, "High bit rate all-optical signal processing in a fiber photonic wire", Optics Express vol.16, 11506-11512 (2008).

[105] V. G. Ta'eed, et al., "Self-phase modulation based integrated optical regeneration in chalcogenide waveguides", IEEE Journal of Selected Topics in Quantum Electronics, vol. 12, no. 3, pp. 360-370, 2006.

[106] L.Fu, M.Rochette, V. Ta'eed, I.C.M.Littler, D.J. Moss and B.J.Eggleton, "Investigation of self-phase modulation based optical regeneration schemes in single mode As2Se3 chalcogenide glass fiber", Optics Express, vol. 13, 7637 (2005).

[107] V.G. Ta'eed et al., "Integrated all-optical pulse regeneration in chalcogenide waveguides", Optics Letters, vol. 30, 2900 (2005).

[108] V.G. Ta'eed et al., "All Optical Wavelength Conversion via Cross Phase Modulation in Chalcogenide Glass Rib Waveguides", Optics Express, vol. 14, 11242 (2006)

[109] V.G. Ta'eed et al., "Self-phase modulation based integrated optical regeneration in chalcogenide waveguides", IEEE Journal of Selected Topics in Quantum Electronics, Vol.12, 360 (2006).

[110] M. Rochette, L.B. Fu, V.G. Ta'eed, I.C.M. Littler, D.J. Moss, B.J. Eggleton, "2R Optical Regeneration: Beyond Noise Compression to BER Reduction", IEEE Journal of Selected Topics in Quantum Electronics, vol. 12, 736 (2006).

[111] V.G. Ta'eed, L.Fu, M.Rochette, I.C. M. Littler, D.J. Moss, B.J. Eggleton, "Error-Free All-Optical Wavelength Conversion in Highly Nonlinear $\mathrm{As}_{2} \mathrm{Se}_{3}$ Chalcogenide Glass Fiber", Optics Express, vol. 14, 10371 (2006).

[112] V.G. Ta'eed, N. Baker, L. Fu, K. Finsterbusch, M.R.E. Lamont, H.C. Nguyen, D.J. Moss, and B.J. Eggleton, Y. Choi, S. Madden, B. Luther-Davies "Ultrafast all-optical chalcogenide glass photonic circuits", Optics Express, vol. 15, 9205 (2007).

[113] C. Monat et al., "Slow light enhanced nonlinear optics in dispersion engineered slow-light silicon photonic crystal waveguides", IEEE Journal of Selected Topics in Quantum Electronics, vol. 16, no. 1, pp. 344-356 (2010).

[114] B. Corcoran, C. Monat, C. Grillet, M.Pelusi, T. P. White, L. O'Faolain, T. F. Krauss, B. J. Eggleton, and D. J. Moss, "Optical signal processing on a silicon chip at $640 \mathrm{~Gb} / \mathrm{s}$ using slow-light", Optics Express, vol. 18, no. 8, 7770-7781 (2010).

[115] V. G. Ta'eed, et al., "Self-phase modulation based integrated optical regeneration in chalcogenide waveguides", IEEE Journal of Selected Topics in Quantum Electronics, vol. 12, no. 3, pp. 360-370, 2006.

[116] M. Shokooh-Saremi, et al., "High performance Bragg gratings in chalcogenide rib waveguides written with a modified Sagnac interferometer: experiment and modeling", Journal of the Optical Society of America B (JOSA B), vol. 23, no. 7, pp. 1323-1331, 2006.

[117] M. R. E. Lamont, et al., "Error-free wavelength conversion via cross phase modulation in $5 \mathrm{~cm}$ of $\mathrm{As}_{2} \mathrm{~S}_{3}$ chalcogenide glass rib waveguide", Electronics Letters, vol. 43, pp. 945-947, 2007.

[118] T.Monro, D.J.Moss, M. Bazylenko, C. Martijn de Sterke, and L. Poladian, "Observation of self-trapping of light in a self written channel in photosensitive glass", Physical Review Letters, vol. 80, 4072 (1998).

[119] T.Ido, H.Sano, D.J.Moss, S.Tanaka, and A.Takai, "Strained InGaAs/InAlAs MQW electroabsorption modulators with large bandwidth and low driving voltage", IEEE Photonics Technology Letters, Vol. 6, 1207 (1994).

[120] E. Ghahramani, D.J. Moss, J.E. Sipe, "Linear and nonlinear optical properties of (GaAs) m/(AlAs) n superlattices", Physical Review B Vol. 43, No. 11, 9269 (1991)

[121] D. J. Moss, et al., "Ultrafast all-optical modulation via two-photon absorption in silicon-insulator waveguides," Electronics Letters, vol. 41, no. 6, pp. 320-321, 2005. DOI:10.1049/el:20058051

[122] M. R. E. Lamont, et al., "Two-photon absorption effects on selfphase-modulation-based 2R optical regeneration," Photonics Technology Letters, vol. 18, no. 10, pp. 1185-1187, 2006 DOI:10.1109/LPT.2006.874718.
[123] A. Tuniz, G. Brawley, D. J. Moss, and B. J. Eggleton, "Two-photon absorption effects on Raman gain in single mode As2Se3 chalcogenide glass fiber," Optics Express, vol. 16, no. 22, pp. 1852418534,2008

[124] J. Wu, L. Jia, Y. Zhang, Y. Qu, B. Jia, and D. J. Moss, "Graphene oxide: versatile films for flat optics to nonlinear photonic chips", Advanced Materials, vol. 32, 2006415, pp.1-29 (2020). DOI:10.1002/adma.202006415.

[125] L. Jia, J. Wu, Y. Yang, Y. Du, B. Jia, D. J. Moss, "Large ThirdOrder Optical Kerr Nonlinearity in Nanometer-Thick $\mathrm{PdSe}_{2} 2 \mathrm{D}$ dichalcogenide Films: Implications for Nonlinear Photonic Devices", ACS Applied Nano Materials, Vol. 3, No. 7, 6876-6883 June 29 (2020). DOI:10.1021/acsanm.0c01239.

[126] L. Jia et al., "BiOBr nanoflakes with strong nonlinear optical properties towards hybrid integrated photonic devices", Applied Physics Letters (APL) Photonics, vol. 4, 090802 (2019).

[127] Y. Zhang et al., "Enhanced Kerr nonlinearity and nonlinear figure of merit in silicon nanowires integrated with 2D graphene oxide films", ACS Applied Materials and Interfaces, vol. 12, no. 29, 33094-33103 June 29 (2020). DOI:10.1021/acsami.0c07852

[128] Y. Qu et al, "Enhanced nonlinear four-wave mixing in silicon nitride waveguides integrated with 2D layered graphene oxide films", Advanced Optical Materials, Vol. 8, No. 21, 2001048 (2020).

[129] J. Wu et al., "Graphene oxide waveguide polarizers and polarization selective micro-ring resonators", Laser and Photonics Reviews, Vol. 13, No. 9, 1900056 (2019).

[130] Y. Yang et al., "Enhanced four-wave mixing in graphene oxide coated waveguides", Applied Physics Letters (APL) Photonics, vol. 3, 120803 (2018).

[131] M. F. Limonov et al., "Fano resonances in photonics," Nat. Photon.., vol. 11, pp. 543-554, Sep. 2017.

[132] E. Kamenetskii, A. Sadreev, and A. Miroshnichenko, "Fano resonances in optics and microwaves: physics and applications," Springer International Publishing, 2018.

[133] E. Miroshnichenko, S. Flach, and Y. S. Kivshar, "Fano resonances in nanoscale structures," Rev. Mod. Phys., vol. 82, no. 3, pp. $2257-$ 2298, Aug. 2010.

[134] C. Grillet et al., "Characterization and modeling of Fano resonances in chalcogenide photonic crystal membranes," Opt. Express, vol. 14, no. 1, pp. 369-376, Jan. 2006.

[135] U. Fano, "Sullo spettro di assorbimento dei gas nobili presso il limite dello spettro d'arco," Il Nuovo Cimento (1924-1942), vol. 12, no. 3, pp. 154-161, Mar. 1935.

[136] U. Fano, "Effects of configuration interaction on intensities and phase shifts," Phys. Rev., vol. 124, no. 6, pp. 1866-1878, Dec. 1961.

[137] L. Stern, M. Grajower, and U. Levy, "Fano resonances and alloptical switching in a resonantly coupled plasmonic-atomic system," Nat. Commun., vol. 5, no. 1, pp. 1-9, Sep. 2014.

[138] Y. Deng et al., "Tunable and high-sensitivity sensing based on Fano resonance with coupled plasmonic cavities," Sci. Rep., vol. 7, no. 1, pp. 1-7, Sep. 2017.

[139] B. S. Luk'yanchuk, A. E. Miroshnichenko, and Y. S. Kivshar, "Fano resonances and topological optics: an interplay of far- and near-field interference phenomena," J. Opt., vol. 15, no. 7, May 2013, Art. no. 073001

[140] B. Peng et al., "What is and what is not electromagnetically induced transparency in whispering-gallery microcavities," Nat. Commun., vol. 5, no. 1, pp. 1-9, Oct. 2014.

[141] Q. Li et al., "Coupled mode theory analysis of mode-splitting in coupled cavity system," Opt. Express, vol. 18, no. 8, pp. 83678382, Apr. 2010.

[142] M. C. Souza et al., "Spectral engineering with coupled microcavities: active control of resonant mode-splitting," Opt. Lett., vol. 40, no. 14, pp. 3332-5, Jul 2015.

[143] L. A. M. Barea et al., "Silicon technology compatible photonic molecules for compact optical signal processing," Appl. Phys. Lett., vol. 103, no. 20, 2013, Art. no. 201102.

[144] L. Zhou, T. Ye, and J. Chen, "Coherent interference induced transparency in self-coupled optical waveguide-based resonators," Opt. Lett., vol. 36, no. 1, pp. 13-15, Jan. 2011.

[145] C. M. Gentry, X. Zeng, and M. A. Popović, "Tunable coupledmode dispersion compensation and its application to on-chip resonant four-wave mixing," Opt. Lett., vol. 39, no. 19, pp. 56895692, Oct. 2014. 
[146] S. Kim et al., "Dispersion engineering and frequency comb generation in thin silicon nitride concentric microresonators," Nat Commun., vol. 8, no. 1, pp. 1-8, Aug. 2017.

[147] Q. Li et al., "Dense wavelength conversion and multicasting in a resonance-split silicon microring," Appl. Phys. Lett., vol. 93, no. 8 Aug. 2008, Art. no. 081113.

[148] M. C. M. M. Souza, "Embedded coupled microrings with highfinesse and close-spaced resonances for optical signal processing," Opt. Express, vol. 22, no. 9, pp. 10430-10438, Apr. 2014.

[149] J. Wu et al., "On-chip tunable second-order differential-equation solver based on a silicon photonic mode-split microresonator," $J$. Lightw. Technol., vol. 33, no. 17, pp. 3542-3549, Sep. 2015.

[150] J. Wu et al., "Passive silicon photonic devices for microwave photonic signal processing," Opt. Commun., vol. 373, pp. 44-52, Aug. 2016.

[151] J. Zhu et al., "On-chip single nanoparticle detection and sizing by mode splitting in an ultrahigh-Q microresonator," Nat. Photon. vol. 4, no. 1, pp. 46-49, Jan. 2010.

[152] A. V. Oppenheim, A. S. Willsky, and S. H. Nawab, "Signals and systems: Pearson New International Edition.” Pearson Education Limited, 2013

[153] C. K. Madsen and J. H. Zhao, "Digital filter concepts for optical filters," in Optical filter design and analysis, pp. 95-164, 1999.

[154] H. Zumbahlen, "Analog filters," in Linear circuit design handbook, H. Zumbahlen Ed. Burlington: Newnes, pp. 581-679, 2008.

[155] J. C. T. Badajos et al., "Reduction of audio noise with lowpass Chebyshev Type II filter simulated using GNU octave," in 2019 IEEE 11th International Conference on Humanoid, Nanotechnology, Information Technology, Communication and Control, Environment, and Management ( HNICEM ), pp. 1-6, 29 Nov.-1 Dec. 2019

[156] S. A. Jadhav et al., "Designing of stepped impedance Butterworth and Chebyshev filters for wireless communication," in 2017 IEEE Applied Electromagnetics Conference (AEMC), pp. 1-2, 19-22 Dec. 2017.

[157] K. Kim et al., "Sinusoidal modeling using elliptic filter for analysis and synthesis of speech signals," in 2006 SICE-ICASE International Joint Conference, pp. 1705-1708, 18-21 Oct. 2006.

[158] K. S. Kumar, B. Yazdanpanah, and P. R. Kumar, "Removal of noise from electrocardiogram using digital FIR and IIR filters with various methods," in 2015 International Conference on Communications and Signal Processing (ICCSP), pp. 0157-0162, 2-4 April 2015.

[159] H. C. Liu and A. Yariv, "Synthesis of high-order bandpass filters based on coupled-resonator optical waveguides (CROWs)," Opt. Express, vol. 19, no. 18, pp. 17653-17668, Aug. 2011.

[160] V. Van, "Synthesis of elliptic optical filters using mutually coupled microring resonators," J. Lightw. Technol., vol. 25, no. 2, pp. 584 590, Feb. 2007.

[161] V. Van, "Advanced microring photonic filter design," in Photonic Microresonator Research and Applications, I. Chremmos, O. Schwelb, and N. Uzunoglu Eds. Boston, MA: Springer US, pp. $115-138,2010$.

[162] C. M. Weinert, "Optical filters in wavelength-division multiplex systems," in Wavelength Filters in Fibre Optics, H. Venghaus Ed. Berlin, Heidelberg: Springer Berlin Heidelberg, pp. 7-16, 2006.

[163] J. Wu et al. "Advanced photonic filters based on cascaded Sagnac loop reflector resonators in silicon-on-insulator nanowires," $A P L$ Photon., vol. 3, no. 4, Apr. 2018, Art. no. 046102.

[164] H. Arianfard et al., "Advanced multi-functional integrated photonic filters based on coupled Sagnac loop reflectors," $J$ Lightw. Technol., pp. 1-9, 2020, Early access: doi 10.1109/JLT.2020.3037559.

[165] J. Wu et al., "Compact on-chip $1 \times 2$ wavelength selective switch based on silicon microring resonator with nested pairs of subrings," Photon. Research, vol. 3, no. 1, pp. 9-14, Feb. 2015.

[166] J. Wu et al., "Micro-ring resonator quality factor enhancement via an integrated Fabry-Perot cavity," APL Photon., vol. 2, no. 5, Apr. 2017, Art. no. 056103.

[167] J. Wu et al., "Nested configuration of silicon microring resonator with multiple coupling regimes," IEEE Photonics Technol. Lett. vol. 25 , no. 6 , pp. 580-583, Feb. 2013.
[168] T. Zhao et al., "Independently tunable double Fano resonances based on waveguide-coupled cavities," Opt. Lett., vol. 44, no. 12, pp. 3154-3157, Jun. 2019.

[169] G. Zhao et al., "Tunable Fano resonances based on microring resonator with feedback coupled waveguide," Opt. Express, vol. 24,pp. 20187-20195, May. 2016.

[170] “G.wdm.1.” http://handle.itu.int/11.1002/1000/11482

[171] B. E. Little et al., "Very high-order microring resonator filters for WDM applications," IEEE Photonics Technol. Lett., vol. 16, no. 10, pp. 2263-2265, Oct. 2004.

[172] F. Xia et al., "Ultra-compact high order ring resonator filters using submicron silicon photonic wires for on-chip optical interconnects," Opt. Express, vol. 15, no. 19, pp. 11934-11941, Sep. 2007.

[173] F. Xia, L. Sekaric, and Y. Vlasov, "Ultracompact optical buffers on a silicon chip," Nat. Photon., vol. 1, no. 1, pp. 65-71, Jan. 2007.

[174] J. K. S. Poon et al., "Designing coupled-resonator optical waveguide delay lines," J. Opt. Soc. Am. B, vol. 21, no. 9, pp. 1665-1673, Sep. 2004.

[175] A. Melloni and M. Martinelli, "Synthesis of direct-coupledresonators bandpass filters for WDM systems," J. Lightw. Technol., vol. 20, no. 2, pp. 296-303, Feb. 2002.

[176] T. Wang et al., "Pulse delay and advancement in SOI microring resonators with mutual mode coupling," J. Lightw. Technol., vol. 27, no. 21, pp. 4734-4743, Nov. 2009.

[177] R. J. Cameron, "General coupling matrix synthesis methods for Chebyshev filtering functions," IEEE Trans. Microw. Theory Tech., vol. 47, no. 4, pp. 433-442, Apr. 1999.

[178] A. M. Prabhu and V. Van, "Realization of asymmetric optical filters using asynchronous coupled-microring resonators," Opt. Express, vol. 15, no. 15, pp. 9645- 9658, Jul. 2007. 